\title{
Hard x-ray Morphological and Spectral Studies of the Galactic Center Molecular Cloud SGR B2: Constraining Past SGR A* Flaring Activity
}

Zhang, Shuo; Hailey, Charles J.; Mori, Kaya; Clavel, Maica; Terrier, Regis; Ponti, Gabriele; Goldwurm, Andrea; Bauer, Franz E.; Boggs, Steven E.; Christensen, Finn Erland

Total number of authors:

18

Published in:

Astrophysical Journal

Link to article, DOI:

10.1088/0004-637x/815/2/132

Publication date:

2015

Document Version

Publisher's PDF, also known as Version of record

Link back to DTU Orbit

Citation (APA):

Zhang, S., Hailey, C. J., Mori, K., Clavel, M., Terrier, R., Ponti, G., Goldwurm, A., Bauer, F. E., Boggs, S. E., Christensen, F. E., Craig, W. W., Harrison, F. A., Hong, J., Nynka, M., Soldi, S., Stern, D., Tomsick, J. A., \& Zhang, W. W. (2015). Hard x-ray Morphological and Spectral Studies of the Galactic Center Molecular Cloud SGR B2: Constraining Past SGR A* Flaring Activity. Astrophysical Journal, 815(2), [132].

https://doi.org/10.1088/0004-637x/815/2/132

\section{General rights}

Copyright and moral rights for the publications made accessible in the public portal are retained by the authors and/or other copyright owners and it is a condition of accessing publications that users recognise and abide by the legal requirements associated with these rights.

- Users may download and print one copy of any publication from the public portal for the purpose of private study or research.

- You may not further distribute the material or use it for any profit-making activity or commercial gain

- You may freely distribute the URL identifying the publication in the public portal 


\title{
HARD X-RAY MORPHOLOGICAL AND SPECTRAL STUDIES OF THE GALACTIC CENTER MOLECULAR CLOUD SGR B2: CONSTRAINING PAST SGR A* FLARING ACTIVITY
}

\author{
Shuo Zhang ${ }^{1}$, Charles J. Hailey ${ }^{1}$, Kaya Mori $^{1}$, Maïca Clavel ${ }^{2}$, Régis Terrier ${ }^{3}$, Gabriele Ponti ${ }^{4}$, Andrea Goldwurm ${ }^{2,3}$, \\ Franz E. Bauer ${ }^{5,6,7}$, Steven E. Boggs ${ }^{8}$, Finn E. Christensen ${ }^{9}$, William W. Craig ${ }^{8,10}$, Fiona A. Harrison ${ }^{11}$, Jaesub Hong ${ }^{12}$, \\ Melania Nynka ${ }^{1}$, Simona Soldi ${ }^{3}$, Daniel Stern ${ }^{13}$, John A. Tomsick ${ }^{8}$, and William W. Zhang ${ }^{14}$ \\ ${ }^{1}$ Columbia Astrophysics Laboratory, Columbia University, New York, NY 10027, USA; shuo@astro.columbia.edu \\ ${ }^{2}$ Service d'Astrophysique/IRFU/DSM, CEA Saclay, F-91191 Gif-sur-Yvette Cedex, France \\ ${ }^{3}$ Unité mixte de recherche Astroparticule et Cosmologie, 10 rue Alice Domon et Léonie Duquet, F-75205 Paris, France \\ ${ }^{4}$ Max-Planck-Institut für extraterrestrische Physik, Giessenbachstrasse 1, D-85748, Garching bei München, Germany \\ ${ }^{5}$ Instituto de Astrofísica, Facultad de Física, Pontificia Universidad Católica de Chile, 306, Santiago 22, Chile \\ ${ }^{6}$ Millennium Institute of Astrophysics, Chile \\ ${ }^{7}$ Space Science Institute, 4750 Walnut Street, Suite 205, Boulder, CO 80301, USA \\ ${ }^{8}$ Space Sciences Laboratory, University of California, Berkeley, CA 94720, USA \\ 9 DTU Space-National Space Institute, Technical University of Denmark, Elektrovej 327, DK-2800 Lyngby, Denmark \\ ${ }^{10}$ Lawrence Livermore National Laboratory, Livermore, CA 94550, USA \\ ${ }^{11}$ Cahill Center for Astronomy and Astrophysics, California Institute of Technology, Pasadena, CA 91125, USA \\ ${ }^{12}$ Harvard-Smithsonian Center for Astrophysics, Cambridge, MA 02138, USA \\ ${ }^{13}$ Jet Propulsion Laboratory, California Institute of Technology, Pasadena, CA 91109, USA \\ ${ }^{14}$ X-ray Astrophysics Laboratory, NASA Goddard Space Flight Center, Greenbelt, MD 20771, USA \\ Received 2015 July 24; accepted 2015 November 7; published 2015 December 21
}

\begin{abstract}
In 2013, NUSTAR observed the Sgr B2 region and for the first time resolved its hard X-ray emission on subarcminute scales. Two prominent features are detected above $10 \mathrm{keV}$ : a newly emerging cloud, G0.66-0.13, and the central 90" radius region containing two compact cores, Sgr B2(M) and Sgr B2(N), surrounded by diffuse emission. It is inconclusive whether the remaining level of Sgr B2 emission is still decreasing or has reached a constant background level. A decreasing X-ray emission can be best explained by the X-ray reflection nebula scenario, where the cloud reprocesses a past giant outburst from Sgr A*. In the X-ray reflection nebula (XRN) scenario, the 3-79 keV Sgr B2 spectrum allows us to self-consistently test the XRN model using both the Fe K $\alpha$ line and the continuum emission. The peak luminosity of the past Sgr $\mathrm{A}^{\star}$ outburst is constrained to $L_{3-79 \mathrm{keV}} \sim 5 \times 10^{38} \mathrm{erg} \mathrm{s}^{-1}$. A newly discovered cloud feature, G0.66-0.13, shows different timing variability. We suggest that it could be a molecular clump located in the Sgr B2 envelope reflecting the same Sgr A* outburst. In contrast, if the Sgr B2 X-ray emission has reached a constant background level, it would imply an origin of lowenergy cosmic-ray (CR) proton bombardment. In this scenario, from the NuSTAR measurements we infer a CR ion power of $d W / d t=(1-4) \times 10^{39} \mathrm{erg} \mathrm{s}^{-1}$ and a CR ionization rate of $\zeta_{\mathrm{H}}=(6-10) \times 10^{-15} \mathrm{H}^{-1} \mathrm{~s}^{-1}$. These measurements can become powerful tools to constrain the GC CR population.
\end{abstract}

Key words: Galaxy: center - ISM: clouds - X-rays: individual (Sgr B2) - X-rays: ISM

\section{INTRODUCTION}

The Galactic center (GC) supermassive black hole Sagittarius $A^{\star}\left(\operatorname{Sgr} A^{\star}\right)$ is the closest such object and is thus an ideal target for investigation of galactic nuclei and their activity cycles (Morris et al. 1999; Ponti et al. 2013). Sgr A* is an underluminous black hole with a bolometric luminosity about $10^{-9}$ times the Eddington luminosity for a $4 \times 10^{6} M_{\odot}$ black hole (Ghez et al. 2008; Gillessen et al. 2009). Its current $\mathrm{X}$-ray quiescent state, with a luminosity of $L_{\mathrm{X}} \sim 10^{33} \mathrm{erg} \mathrm{s}^{-1}$ (Baganoff et al. 2003), is punctuated by flares up to a few times $10^{35} \mathrm{erg} \mathrm{s}^{-1}$ (e.g., Baganoff et al. 2001; Porquet et al. 2008; Nowak et al. 2012), during which the bolometric luminosity is still orders of magnitude lower than its Eddington luminosity. Hard X-rays up to $\sim 79 \mathrm{keV}$ have also been detected from the flares by NUSTAR (Barrière et al. 2014). Whether it has ever experienced more substantial increases in activity as observed in low-luminosity active galactic nuclei (AGNs) is still under discussion.

Indication of such past activity of Sgr $\mathrm{A}^{\star}$ has come from Galactic center molecular clouds (GCMCs). In the populous Central Molecular Zone (CMZ; Morris \& Serabyn 1996), Sgr B2 is the densest and most massive molecular cloud, with a total mass of $M \sim 6 \times 10^{6} M_{\odot}$ and a high column density of $N_{\mathrm{H}} \sim 10^{24} \mathrm{~cm}^{-2}$ through the cloud (Lis \& Goldsmith 1990). It has complicated substructures, including compact star-forming cores Sgr B2(M), Sgr B2(N), and Sgr B2(S) (e.g., Benson \& Johnston 1984; Sato et al. 2000; Etxaluze et al. 2013). In an extremely simplified picture, its density profile can be described as a dense core with a radius of $0.15-0.3 \mathrm{pc}\left(2^{\prime \prime}-\right.$ $4^{\prime \prime}$ assuming the Sgr B2 distance of $7.9 \pm 0.8 \mathrm{kpc}$; Reid et al. 2009) and an $\mathrm{H}_{2}$ density of (3-9) $\times 10^{6} \mathrm{~cm}^{-3}$, surrounded by an envelope extended to $\sim 5 \mathrm{pc}(\sim 2$ '.2) with a density of $10^{4}-10^{5} \mathrm{~cm}^{-3}$ and a larger diffuse component reaching $\sim 22.5 \mathrm{pc}$ with a roughly constant density of $\sim 10^{3} \mathrm{~cm}^{-3}$ (Lis \& Goldsmith 1990; de Vicente et al. 1997).

Sgr B2 is the first GCMC from which a strong $6.4 \mathrm{keV} \mathrm{Fe}$ $\mathrm{K} \alpha$ line was discovered (Koyama et al. 1996). ASCA detected this significant line feature with an equivalent width (EW) of about $1 \mathrm{keV}$, which was later confirmed by Chandra, Suzaku, and XMM-Newton observations (Murakami et al. 2001; Koyama et al. 2007; Terrier et al. 2010). Time variability of the $\mathrm{Sgr} \mathrm{B} 2 \mathrm{Fe} \mathrm{K} \alpha$ line was revealed by years of monitoring by different instruments. The $6.4 \mathrm{keV}$ line flux began declining in 2001, decreased by a factor of $\sim 0.4$ by 2005 (Inui et al. 2009), 
and further decreased by a factor of $0.39 \pm 0.04$ from 2005 to 2009 (Nobukawa et al. 2011). The flux and morphology change of the X-ray emission is also observed from other GCMCs. Interestingly, the clouds exhibit $\mathrm{Fe} \mathrm{K} \alpha$ line variability in different ways, some rising and others decreasing, and an emission peak is detected in the "Bridge" structure (centered on R.A. $=17^{\mathrm{h}} 46^{\mathrm{m}} 06^{\mathrm{s}} .9$, decl. $=-28^{\circ} 54^{\prime} 31^{\prime \prime} 4$, J2000) with a timescale as short as 2 yr (Muno et al. 2007; Ponti et al. 2010; Capelli et al. 2012; Clavel et al. 2013).

Reflection of incoming X-rays by cold molecular material is a natural explanation of the observed X-ray emission. In the $\mathrm{X}$-ray reflection nebula (XRN) model, K-shell photoionization and the subsequent fluorescence produce a strong $\mathrm{Fe} \mathrm{K} \alpha$ line with an equivalent width $\mathrm{EW} \geqslant 1 \mathrm{keV}$, while a competition between Compton scattering of high-energy photons and photoelectric absorption of low-energy photons gives rise to a Compton reflection hump around 20-30 keV (Sunyaev et al. 1993; Koyama et al. 1996; Sunyaev \& Churazov 1998). An embedded or nearby transient X-ray source was ruled out as an illuminating source, since no transient source has been sufficiently bright $\left(L \geqslant 10^{37} \mathrm{erg} \mathrm{s}^{-1}\right)$ for several years since 1993 within or close to Sgr B2 (e.g., Revnivtsev et al. 2004; Terrier et al. 2010). Sgr $A^{\star}$ was proposed to be the likely source illuminating Sgr B2. A major outburst from Sgr $A^{\star}$, with a luminosity of a few times $10^{39} \mathrm{erg} \mathrm{s}^{-1}$ lasting more than $10 \mathrm{yr}$ and ending a few hundred years ago, would explain the Sgr B2 emission (Koyama et al. 1996; Terrier et al. 2010). This hypothesis is reinforced by the discovery of a superluminal Fe $\mathrm{K}$ echo from the "Bridge," which points to propagation of an event far away from the clouds (Ponti et al. 2010). However, the story gets complicated by different $\mathrm{Fe} \mathrm{K} \alpha$ line variability detected from GCMCs, which cannot be explained with a single outburst from Sgr $\mathrm{A}^{\star}$. The strong and fast variation of the $\mathrm{Fe} \mathrm{K} \alpha$ line flux in the "Bridge" region requires a $2 \mathrm{yr}$ peaked outburst with luminosity of at least $10^{39} \mathrm{erg} \mathrm{s}^{-1}$, while the slower $\mathrm{Fe} \mathrm{K} \alpha$ line behavior detected in other clouds suggests a second flare with a longer duration (Clavel et al. 2013).

The propagation of cosmic-ray (CR) particles within the molecular clouds is an alternative explanation. Low-energy cosmic-ray electrons (LECRe) and protons (LECRp) can both produce hard X-rays and $\mathrm{Fe} \mathrm{K} \alpha$ emission (Valinia et al. 2000; Dogiel et al. 2009). The Sgr B2 Fe K $\alpha$ flux variation timescale of $\sim 10 \mathrm{yr}$ is too short compared to the Coulomb cooling time of $\sim 100 \mathrm{MeV}$ protons invoked in the LECRp scenario, thus ruling it out as a major contributor to the fast-changing $\mathrm{Fe} \mathrm{K} \alpha$ emission of Sgr B2 (Terrier et al. 2010). However, LECRp could be a major contributor to the constant background level of the Sgr B2 Fe K $\alpha$ emission, which could be detectable once the reflected X-ray emission completely fades. Dogiel et al. (2009) estimated that LECRp could contribute to about $15 \%$ of the observed maximum $\mathrm{Fe} \mathrm{K} \alpha$ flux obtained around 2000. In the LECRe scenario, a fast variation can be produced. Even if LECRe seems to successfully explain the X-ray emission from several GCMCs (Yusef-Zadeh et al. 2007a, 2013), it meets challenges for most of the Fe K $\alpha$ bright structures (Dogiel et al. 2013 , 2014). In the case of Sgr B2, the CR electron energy required to produce the cloud $\mathrm{X}$-ray emission is as high as the bolometric luminosity of the entire cloud (Revnivtsev et al. 2004). By applying the LECRe model to their observation data, Terrier et al. (2010) derived the required metallicity of $Z / Z_{\odot} \sim 3.1$, which is much higher than current measurements of the GC metallicity ranging from slightly higher than solar (Cunha et al. 2008; Davies et al. 2009) to twice solar (Giveon et al. 2002). In contrast, the metallicity required by the XRN scenario is $Z / Z_{\odot} \sim 1.3$, matching well with the GC metallicity measurements. However, even if LECRe is not the dominant process in the GC and particularly in Sgr B2, we cannot exclude that, in specific regions, the LECRe process contributes to the background level of the $\mathrm{Fe} \mathrm{K} \alpha$ emission.

Though the GCMCs have been studied extensively below $10 \mathrm{keV}$, the investigation of the continuum emission extending beyond $10 \mathrm{keV}$ has been limited. The hard X-rays from the Sgr B2 region were first detected by GRANAT/ART-P (Sunyaev et al. 1993) and then by INTEGRAL/IBIS in 2004 (Revnivtsev et al. 2004). In the XRN scenario, Terrier et al. (2010) derived an illuminating source spectral index of $\Gamma \sim 2$ with combined XMM-Newton and INTEGRAL/IBIS spectra. Years of monitoring with INTEGRAL/IBIS reveal that the hard X-ray emission decreases by a factor of 0.4 from 2003 to 2009 (Terrier et al. 2010). Nevertheless, INTEGRAL/IBIS was not able to resolve the hard X-ray emission. The accuracy of the hard X-ray luminosity was limited by the unknown distribution of the emission.

Hard X-ray observations are crucial to better constrain the origin of the GCMC nonthermal X-ray emission. With unprecedented spatial and spectral resolution in the $10-79 \mathrm{keV}$ band, NUSTAR observed GCMCs in 2013 as part of the Galactic plane survey campaign, including the Sgr B2 region. For the first time, $N U S T A R$ resolved its hard X-ray morphology and obtained a broadband spectrum from a single instrument. In Section 2, we introduce the observations and data reduction for NuSTAR and XMM-Newton data used for the following analysis. We present the morphology of the central $90^{\prime \prime}$ radius region of $\mathrm{Sgr} \mathrm{B} 2$ and the newly discovered cloud feature G0.66-0.13 in Section 3, their time variability in Section 4, and their spectroscopy in Section 5. Based on these observational results, we discuss their implications on both XRN and LECR scenarios in Section 6.

\section{OBSERVATION AND DATA REDUCTION}

\subsection{NuSTAR Data}

The NUSTAR observatory operates in the broad X-ray energy band from 3 to $79 \mathrm{keV}$ (Harrison et al. 2013). Sgr B2 was observed by NUSTAR in 2013 October in two $25 \%$ overlapping pointings, with a total exposure time of $293.7 \mathrm{ks}$ (See Table 1).

In both observations, the Sgr B2 region was imaged with the two co-aligned X-ray telescopes, with corresponding focal plane modules FPMA and FPMB, providing an angular resolution of 58" half-power diameter (HPD) and 18" FWHM over the 3-79 keV X-ray band, with a characteristic spectral resolution of $400 \mathrm{eV}$ (FWHM) at $10 \mathrm{keV}$. The nominal reconstructed NUSTAR astrometry is accurate to $8^{\prime \prime}(90 \%$ confidence level; Harrison et al. 2013). The data were reduced and analyzed using the NUSTAR Data Analysis Software NuSTARDAS v. 1.3.1 and HEASOFT v. 6.13 and then filtered for periods of high instrumental background due to South Atlantic Anomaly (SAA) passages and known bad/noisy detector pixels.

The NuSTAR detectors are not completely shielded from incident X-rays that do not go through the optics, which is referred to as stray light. Bright $X$-ray sources within $\sim 1^{\circ}-5^{\circ}$ of the NUSTAR field of view can significantly contaminate one 
Table 1

NUSTAR and XMM-Newton Observations of Sgr B2

\begin{tabular}{lccr}
\hline Instrument & $\begin{array}{c}\text { Observation } \\
\text { ID }\end{array}$ & $\begin{array}{c}\text { Start Time } \\
(\mathrm{UTC})\end{array}$ & $\begin{array}{c}\text { Exposure } \\
(\mathrm{ks})\end{array}$ \\
\hline NuSTAR & 40012018002 & 2013 Oct 22T16:56:07 & 142.2 \\
NuSTAR & 40012019001 & 2013 Oct 25T22:31:07 & 151.6 \\
\hline XMM-Newton & 0112971501 & 2001 Apr 01T00:25:11 & 9.2 \\
XMM-Newton & 0030540101 & 2002 Sep 09T11:11:26 & 19.3 \\
XMM-Newton & 0203930101 & 2004 Sep 04T02:53:45 & 48.5 \\
XMM-Newton & 0694640601 & 2012 Sep 06T10:56:15 & 66.6 \\
XMM-Newton & 0694641301 & 2012 Sep 16T18:34:18 & 72.9 \\
XMM-Newton & 0694641401 & 2012 Sep 30T19:39:50 & $58.3^{\mathrm{a}}$ \\
\hline
\end{tabular}

Note.

a In the observation 0694641401, the exposure time is $58.3 \mathrm{ks}$ for Sgr B2 and $44.8 \mathrm{ks}$ for G0.66-0.13, as for the latter there are no MOS1 data available.

or both of the detector planes. The contaminated detector pixels can be removed based on a numerical model that fully takes into account the telescope geometry (Krivonos et al. 2014). Thus, to make a stray-light-free mosaic, we used this model to remove stray-light patterns from both FPMA and FPMB detectors by flagging the contaminated detector pixels as bad, when processing with the NuSTARDAS pipeline. As a result, stray light from the X-ray sources SLX 1744-299 and 1E 1740.7-2942 was removed from FPMA detectors, and that from GX 3+1 and SLX 1735-269 was removed from FPMB detectors. We then registered these stray-light-free images with the brightest point sources available in individual observations. As a result, the astrometry is improved to $\sim 4^{\prime \prime}$. Lastly, we combined the exposure-corrected images in different energy bands (Figure 1).

On the other hand, removing the stray-light patterns is not necessary for spectral extraction. The stray-light background does not significantly change from one sky pointing to another, provided that they are separated by no more than $10^{\prime}-20^{\prime}$ (Krivonos et al. 2014). Therefore, for the Sgr B2 core region detected in one observation, we extracted the background from the same detector region in the other observation. This was our motivation for using two $25 \%$ overlapping sky pointings. This background subtraction method has been applied to many sources detected by NuSTAR suffering from stray light (e.g., Krivonos et al. 2014; Zhang et al. 2014) and is proven to subtract mild stray-light contamination properly.

Owing to the extremely bright stray-light contamination within $90^{\prime \prime}$ of Sgr B2 on FPMB, we used FPMA data only for the Sgr B2 core spectrum. From the selected data sets, we extracted source spectra from a circular region of $90^{\prime \prime}$ radius centered on Sgr B2 (R.A. $=17^{\mathrm{h}} 47^{\mathrm{m}} 20.4$, decl. $=$ $-28^{\circ} 23^{\prime} 07$ !' 0, J2000). In this way, we had two sourcebackground pairs with the Sgr B2 core detected on FPMA in observations 40012018002 and 40012019001 . We combined the two FPMA source spectra and their associated response files and background spectra. The resultant spectrum was grouped such that the detection significance in each data bin is at least $3 \sigma$. The confidence level for all the error bars reported in this paper is $90 \%$.

Another cloud feature, G0.66-0.13 (R.A. $=17^{\mathrm{h}} 47^{\mathrm{m}} 41^{\mathrm{s}} .5$, decl. $=-28^{\circ} 26^{\prime} 23^{\prime \prime} 0$, J2000; Ponti et al. 2014), is only captured in the second observation (obsID 4001201901) and avoids the stray light from GX $3+1$ on FPMB. We thus used both FPMA and FPMB from the second observation for its spectral analysis. The spectra were combined and grouped with the same method as for the central $90^{\prime \prime}$ region.

\subsection{XMM-Newton Data}

We collected and analyzed all the XMM-Newton data available in the archive covering the Sgr B2 and G0.66-0.13 regions. This includes observations performed in 2000, 2001, 2002, 2004, and 2012. The 2000 data were excluded owing to low effective exposure and poor statistics, while for the other years we used all the available observations. The list of $X M M$ Newton observations is presented in Table 1, along with the total EPIC-pn-equivalent exposure times (i.e., computed assuming a 0.4 ratio between the effective areas of the MOS and pn detectors). For each selected observation we extracted the spectra from all available EPIC instruments using the XMM-Newton Extended Source Analysis Software (ESAS; Snowden et al. 2008) distributed with version 12.0.1 of the XMM-Newton Science Analysis Software. For each exposure, calibrated event files were produced with the tasks epchain and emchain and filtered with pn-filter and mos-filter in order to exclude the time intervals affected by soft proton contamination. The spectra were then extracted with the ESAS mosspectra and pn-spectra scripts and rebinned to have at least 30 counts in each bin to apply chi-square statistics. The quiescent component of the EPIC internal particle background (QPB) was estimated using archival observations provided within the ESAS database and taken with the filter wheel closed.

\section{SPATIAL DISTRIBUTION OF THE HARD X-RAY EMISSION AND THE FE K $\alpha$ LINE EMISSION}

Figure 1 shows the resultant $17^{\prime} \times 11^{\prime} \mathrm{NuSTAR}$ sky mosaics of the Sgr B2 region in the $3-40 \mathrm{keV}, 3-10 \mathrm{keV}, 6.2-6.6 \mathrm{keV}$, and $10-40 \mathrm{keV}$ bands. The $3-40 \mathrm{keV}$ image shows that two features are significantly detected: the central $90^{\prime \prime}$ of Sgr B2 and a newly discovered cloud feature, G0.66-0.13, whose $6.4 \mathrm{keV} \mathrm{Fe} \mathrm{K} \alpha$ emission turned bright in 2012 as revealed by the XMM-Newton data (R. Terrier et al. 2015, in preparation). The green circle of $90^{\prime \prime}$ radius outlines the central region of Sgr B2, corresponding to $3.4 \pm 0.3 \mathrm{pc}$ with a cloud distance of $7.9 \pm 0.8 \mathrm{kpc}$ (Reid et al. 2009). The green ellipse outlines the cloud feature G0.66-0.13, with a semimajor axis of $130^{\prime \prime}$ $(4.9 \pm 0.5 \mathrm{pc})$ and a semiminor axis of $76^{\prime \prime}(2.9 \pm 0.3 \mathrm{pc})$. G0.66-0.13 is $\sim 14 \mathrm{pc}$ away from the center of Sgr B2 in the projected plane. Sgr B2 and G0.66-0.13 are both about 100 pc away from $\mathrm{Sgr} \mathrm{A}^{\star}$ in the projected plane. The lower-energy $3-10 \mathrm{keV}$ image is also shown to compare with previous observations of Sgr B2 by Chandra, XMM-Newton, and other imaging observatories. The $6.2-6.6 \mathrm{keV}$ band image with continuum emission subtracted shows the $\mathrm{Fe} \mathrm{K} \alpha$ line emission morphology of the Sgr B2 region. The 10-40 keV band image provides the line-free continuum emission morphology. All the images are scaled individually to illustrate the morphology of major features. The images are overlaid with $6.4 \mathrm{keV}$ line emission contours. The contours are made from the 2012 image of the XMM-Newton CMZ scan in the $6.28-6.53 \mathrm{keV}$ band, from which the continuum emission (estimated assuming a power-law model between two adjacent bands) has been subtracted (Ponti et al. 2014, 2015).

The $10-40 \mathrm{keV}$ image demonstrates that the high-energy X-ray $(\geqslant 10 \mathrm{keV})$ morphology of Sgr B2 is resolved at subarcminute 

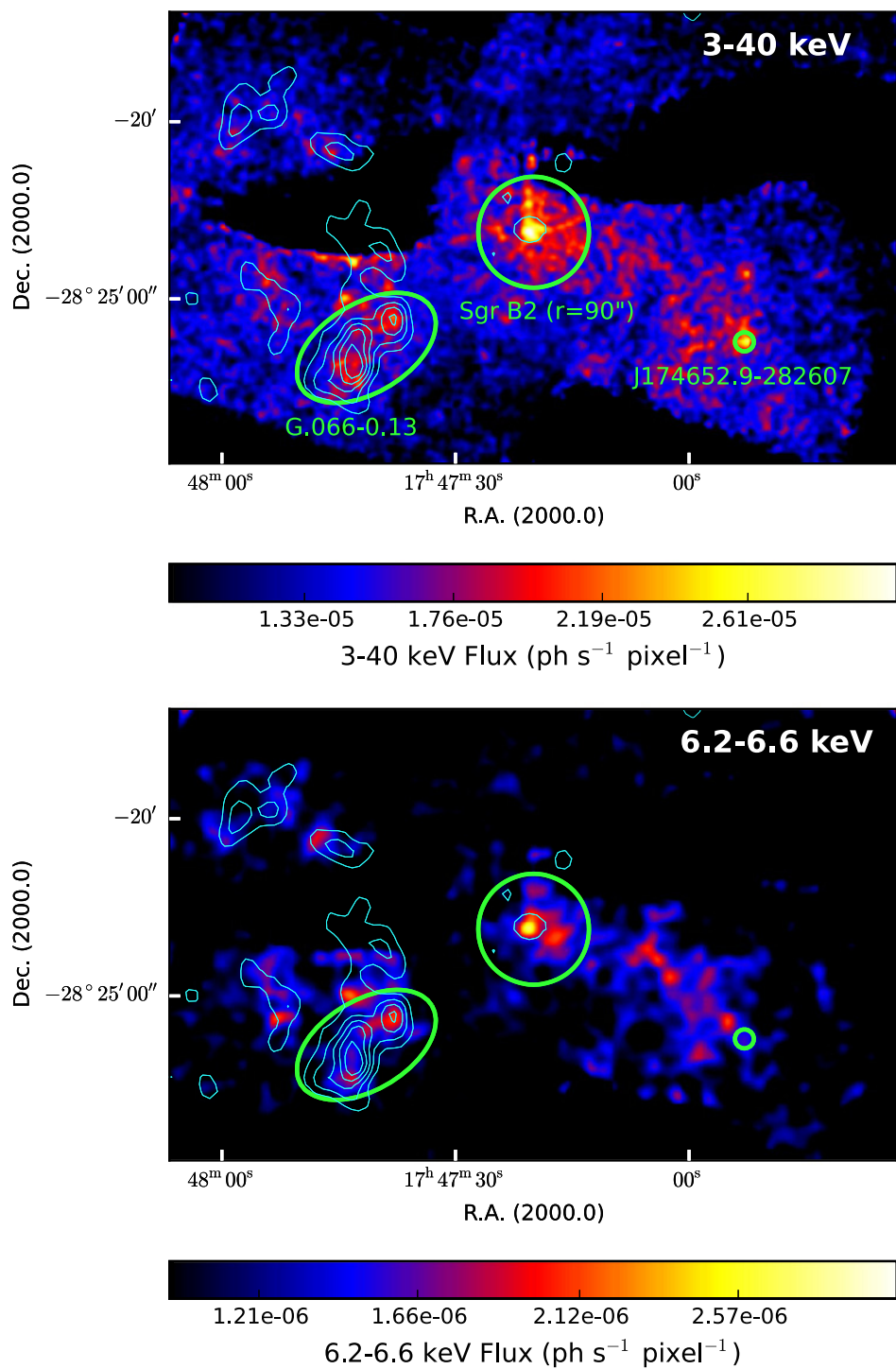
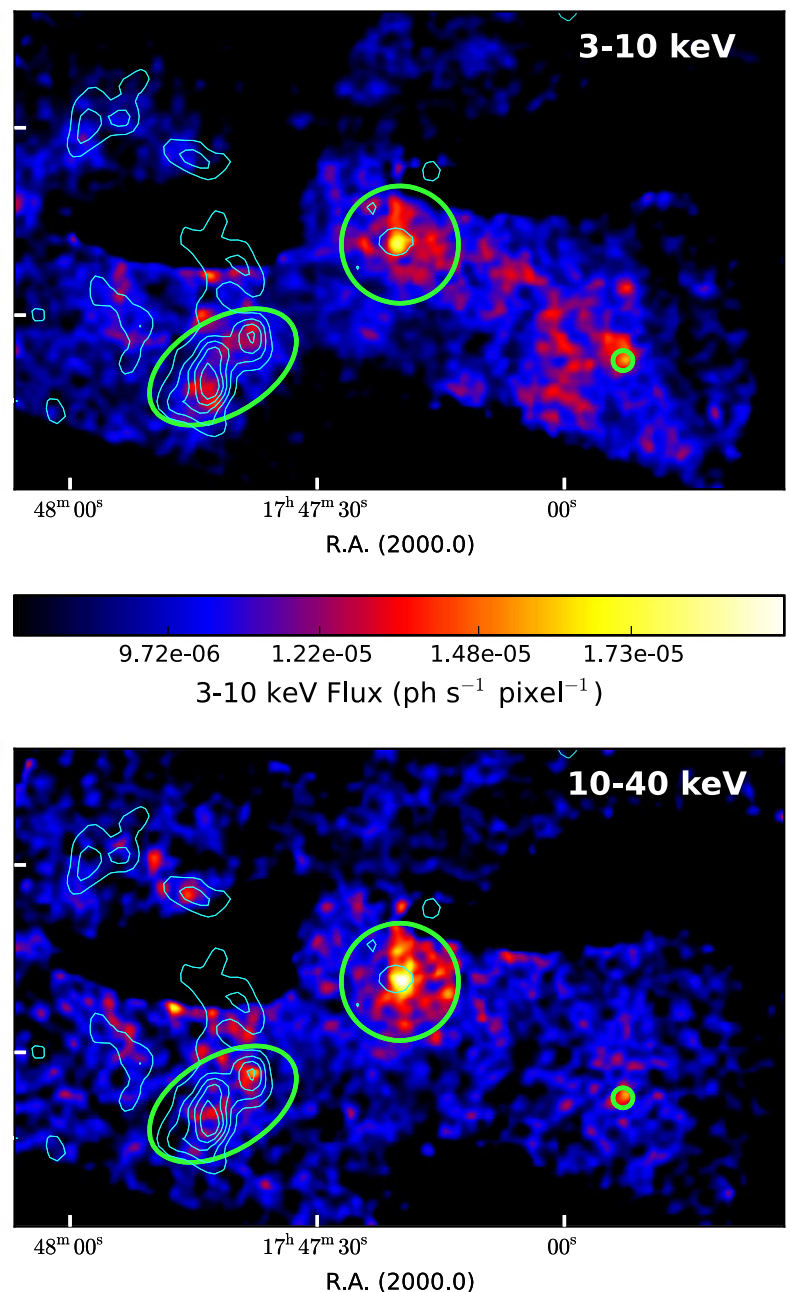

R.A. $(2000.0)$

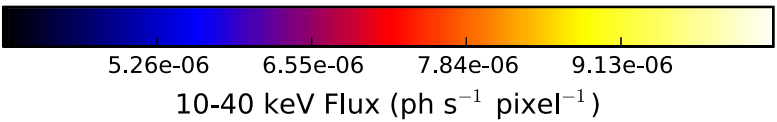

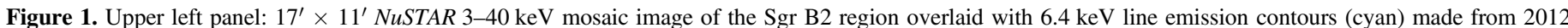

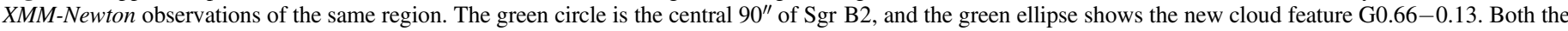

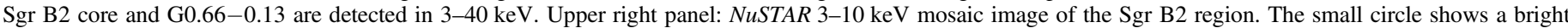

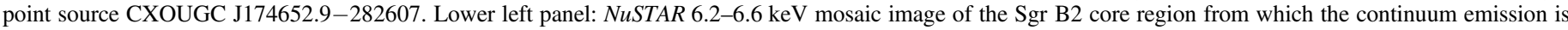

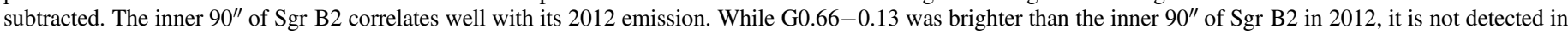

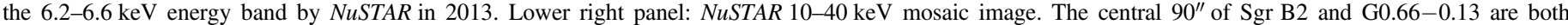

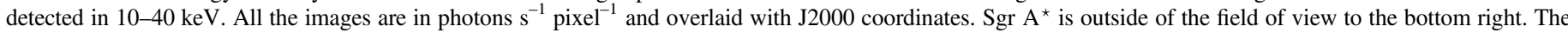

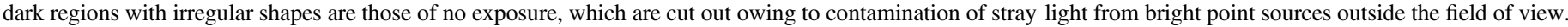

scales for the first time. It clearly reveals substructures of Sgr B2 and proves that the X-ray emission in this energy band is extended (Figure 1). Both the central 90" of Sgr B2 and G0.66 -0.13 are significantly detected above $10 \mathrm{keV}$. G0.66-0.13 shows two bright cores separated by about $100^{\prime \prime}$, well correlated with its $6.4 \mathrm{keV}$ line contour. The size of each peak is $\sim 20^{\prime \prime}$. The $10-40 \mathrm{keV}$ surface brightness of the whole G0.66-0.13 region is $(3.5 \pm 0.8) \times 10^{-6}$ photons $\mathrm{cm}^{-2} \mathrm{~s}^{-1} \operatorname{arcmin}^{-2}$. Within the central $90^{\prime \prime}$ region of Sgr B2, the $10-40 \mathrm{keV}$ emission peaks at the center, coinciding with the compact star-forming core Sgr B2(M), with a surface brightness of $(1.6 \pm 0.1) \times$ $10^{-5}$ photons $\mathrm{cm}^{-2} \mathrm{~s}^{-1} \operatorname{arcmin}^{-2}$ for the central $25^{\prime \prime}$ radius region. It is detected at the $\sim 17 \sigma$ level and likely to be the hard X-ray counterpart of Sgr B2(M). The right panel of Figure 2 shows the zoomed-in $7^{\prime} \times 5^{\prime}$ image of the central region in
10-40 keV. Besides Sgr B2(M), X-ray emission around the additional compact core Sgr B2(N), about 50" north of Sgr B2 (M), is also detected $(\sim 5 \sigma)$. The zoomed-in images in Figure 2 are overlaid with the source regions of Sgr B2(M) (R.A. $=17^{\mathrm{h}} 47^{\mathrm{m}} 20 \mathrm{~s} .30, \quad$ decl. $=-28^{\circ} 23^{\prime} 04^{\prime \prime}$ 01， J2000) and Sgr B2(N) (R.A. $=17^{\mathrm{h}} 47^{\mathrm{m}} 20.30, \quad$ decl. $=-28^{\circ} 23^{\prime} 04^{\prime \prime}$. 01 , J2000) defined from submillimeter band observations by Herschel (Etxaluze et al. 2013). X-ray emission from a third compact core, Sgr B2(S), south of Sgr B2(M), is not detected in $10-40 \mathrm{keV}$. The surrounding regions show lower surface brightness, with the western half of the annulus from $25^{\prime \prime}$ to $90^{\prime \prime}$ brighter than the eastern half. The $10-40 \mathrm{keV}$ morphology strongly resembles the optical depth map at $250 \mu \mathrm{m}$, which indicates the local column density, derived from submillimeter continuum emission (Etxaluze et al. 2013). In the $250 \mu \mathrm{m}$ optical depth map, 

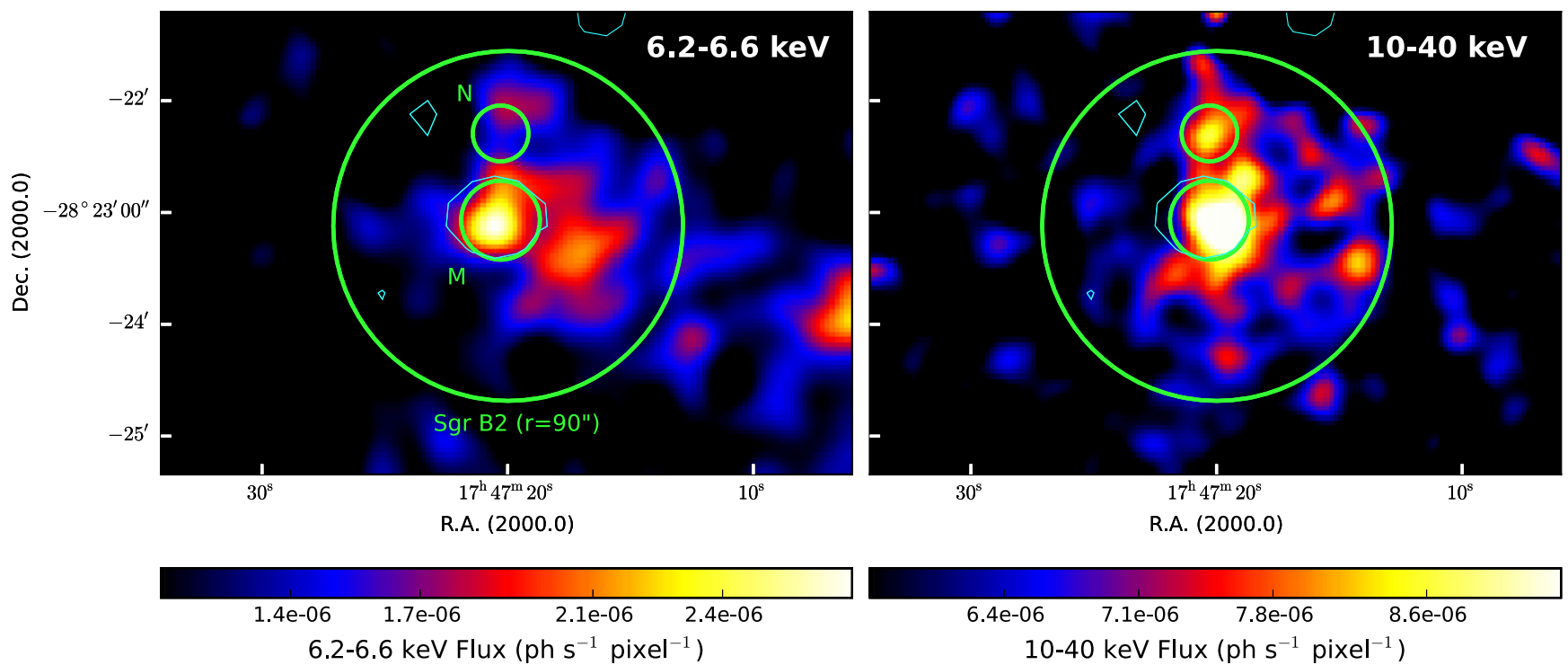

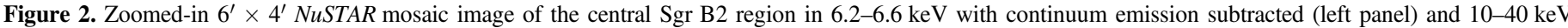

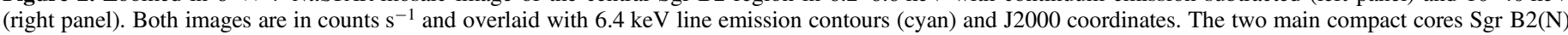

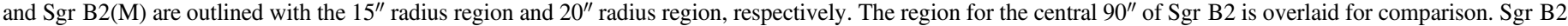
(M) is detected in both energy bands, while Sgr B2(N) is only detected above $10 \mathrm{keV}$.

Sgr B2(M) and Sgr B2(N) have the highest optical depth at $250 \mu \mathrm{m} \quad\left(\tau_{250 \mu \mathrm{m}}>1\right)$, while the surrounding areas have gradually lower $\tau_{250 \mu \mathrm{m}}$, with the western region higher than the eastern. It suggests that the hard X-ray continuum emission traces the local column density of the cloud material.

In $6.2-6.6 \mathrm{keV}$, only the main compact core $\mathrm{Sgr} \mathrm{B} 2(\mathrm{M})$ is significantly detected. Sgr B2(N) is not detected at $6.4 \mathrm{keV}$, nor in the $3-10 \mathrm{keV}$ range (left panel of Figure 2). This could be due to higher local absorption. The $\mathrm{Fe} \mathrm{K} \alpha$ line emission is not detected from the G0.66-0.13 region. This is a dramatic change from its $2012 \mathrm{Fe} \mathrm{K} \alpha$ line morphology, represented by the cyan contours, where G0.66-0.13 was brighter than the Sgr B2 core region. This indicates that the G0.66-0.13 Fe K $\alpha$ emission has a short lifetime ( $e$-folding decay time) of about $1 \mathrm{yr}$.

Finally, we note that the small white circle with $16^{\prime \prime}$ radius illustrates a bright point source CXOUGC J174652.9-282607, which is registered in the Chandra point-source catalog (Muno et al. 2009). The source is detected up to $\sim 40 \mathrm{keV}$ by $N u S T A R$ (R.A. $=17^{\mathrm{h}} 47^{\mathrm{m}} 52^{\mathrm{s}} .968, \quad$ decl. $=-28^{\circ} 26^{\prime} 07^{\prime \prime} \cdot 37$, J2000) and will be reported in another work (J. Hong et al. 2015, in preparation).

\section{TIME VARIABILITY OF CENTRAL SGR B2 AND G0.66-0.13}

With the XMM-Newton data in 2001, 2002, 2004, and 2012 and the most recent $2013 \mathrm{NuSTAR}$ observation of Sgr B2, we obtained the observed $6.4 \mathrm{keV}$ line flux for the central $90^{\prime \prime}$ radius region of $\mathrm{Sgr} \mathrm{B} 2$ using a simple model wabs* (apec+wabs*pow+gauss+gauss) (see Section 5.1.1). The cross-calibration factor between NuSTAR and XMMNewton is less than $10 \%$ (Madsen et al. 2015); thus, the systematic errors in flux measurements between the two instruments are smaller than our statistical errors. All the model parameters are fixed to be the same for all the years, while the normalizations for the power-law and Gaussian components are left free. The fit is satisfactory with $\chi_{\nu}^{2}=1.0$

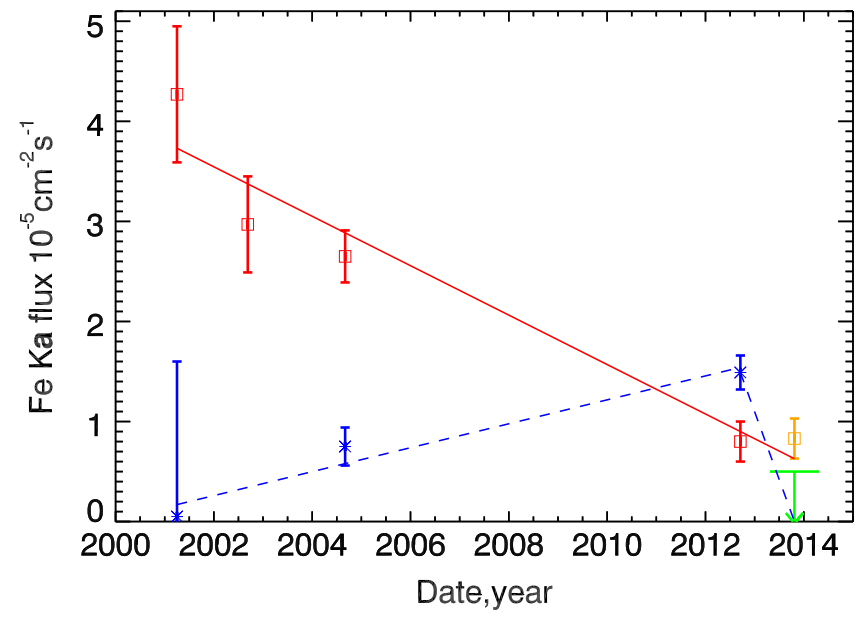

Figure 3. Time variability of the $\mathrm{Fe} \mathrm{K} \alpha$ line for the central $90^{\prime \prime}$ of Sgr B2 and the additional cloud feature G0.66-0.13. The Sgr B2 absorbed $6.4 \mathrm{keV}$ line flux was measured in 2001, 2002, 2004, and 2012 by XMM-Newton (red squares) and in 2013 by NuSTAR (orange square). The Fe $\mathrm{K} \alpha$ line flux shows a clear decay of up to $80 \%$ of the measured Sgr B2 flux from 2001 to 2013. A chi-square hypothesis test favors a linearly decreasing flux at a $6.7 \sigma$ level; the red line shows the best-fit linear decay model with a slope of $s=-0.25 \times 10^{-5}$ photons $\mathrm{cm}^{-2} \mathrm{~s}^{-1} \mathrm{yr}^{-1}$. The absorbed $6.4 \mathrm{keV}$ line flux of G0.66-0.13 was measured in 2001, 2004, and 2012 by XMM-Newton (blue stars) and in 2013 by $N u S T A R$ (green upper limit). The G0.66-0.13 Fe K $\alpha$ emission experienced an increase before 2012 and a fast decay from 2012 to 2013. The blue line shows the best-fit linearly increasing model with a slope of 0.12 . Yet in 2013 the $6.4 \mathrm{keV}$ line was not significantly detected by NUSTAR.

for dof $=517$. The resultant absorbed flux change of the central 90" of Sgr B2 over time is shown in Figure 3 with square data points. The $6.4 \mathrm{keV}$ line flux shows a clear decreasing trend from 2001 to 2013, which can be fit with a linear decay model with a slope of $s=-0.25 \times$ $10^{-5}$ photons $\mathrm{cm}^{-2} \mathrm{~s}^{-1} \mathrm{yr}^{-1}$. The varying flux is preferred at a $\sim 7 \sigma$ level over a flat light curve. In 2013 the observed $6.4 \mathrm{keV}$ line flux is down to $(0.83 \pm 0.21) \times 10^{-5}$ photons $\mathrm{cm}^{-2} \mathrm{~s}^{-1}$, which corresponds to about $20 \%$ of the 2001 observed flux. 
The $6.4 \mathrm{keV}$ line flux decrease rate is compatible with previous works showing that the $6.4 \mathrm{keV}$ line emission of a more extended Sgr B2 region in 2005 is about $60 \%$ that of 2000 (Inui et al. 2009). The lifetime of the X-ray photons is $t \sim 11 \mathrm{yr}$, which agrees well with the $\sim 11 \mathrm{yr}$ light-crossing time for the central 90", with a distance of $7.9 \mathrm{kpc}$ (Reid et al. 2009). Although the 2001-2013 light curve shows a decreasing trend, we note that the $2013 \mathrm{Fe} \mathrm{K} \alpha$ emission is at the same level as that of 2012. With limited statistics, it is not conclusive whether the Sgr B2 Fe K $\alpha$ emission was still decreasing in 2013 or it had reached a constant background emission level. Future observation of the Sgr B2 region will constrain its timing variability.

The 7 yr monitoring of Sgr B2 by INTEGRAL/IBIS reveals that the hard X-ray continuum emission flux decreases with a similar trend to the $6.4 \mathrm{keV}$ emission, both decaying up to $40 \%$ from 2003 to 2009 (Terrier et al. 2010). This decay profile predicts that the hard X-ray emission in 2013 reaches $\sim 30 \%$ that of 2001 . The $10-40 \mathrm{keV}$ flux of the central $90^{\prime \prime}$ of Sgr B2 measured by NuSTAR in 2013 is $F_{10-40 \mathrm{keV}}=(1.9 \pm 0.2) \times 10^{-12} \mathrm{erg} \mathrm{cm}^{-2} \mathrm{~s}^{-1}$. Therefore, the extrapolated $10-40 \mathrm{keV}$ flux of the central Sgr B2 region in 2001 is $F_{10-40 \mathrm{keV}} \sim 6.3 \times 10^{-12} \mathrm{erg} \mathrm{cm}^{-2} \mathrm{~s}^{-1}$ based on the hard X-ray decay profile. To verify the estimated flux in 2001, we extrapolated the 2001 XMM-Newton spectrum into higher energies and derived the $10-40 \mathrm{keV}$ flux of the central $90^{\prime \prime}$ in 2001 to be $F_{10-40 \mathrm{keV}}=(6 \pm 2) \times 10^{-12} \mathrm{erg} \mathrm{cm}^{-2} \mathrm{~s}^{-1}$, which matches with the 2001 flux value extrapolated based on NUSTAR measurements. This will be used to derive the luminosity of the primary source in Section 6.1.

The newly discovered cloud feature G0.66-0.13, 6' (14 pc) from the central Sgr B2 region in the projected plane, was not significantly brighter than surrounding regions at $6.4 \mathrm{keV}$ until 2012. We derived its $6.4 \mathrm{keV}$ line flux from XMM-Newton observations in 2001, 2004, and 2012 (stars in Figure 3). The 2002 XMM-Newton observation has very poor statistics for this region and thus was not used. The 2001 flux is poorly constrained to $F_{6.4 \mathrm{keV}}=0.05 \times 10^{-5}$ photons $\mathrm{cm}^{-2} \mathrm{~s}^{-1}$ with an upper limit of $1.60 \times 10^{-5}$ photons $\mathrm{cm}^{-2} \mathrm{~s}^{-1}$. The 2001 and $2004 \mathrm{Fe} \mathrm{K} \alpha$ emission of G0.66-0.13 remained at the same level as the surrounding regions. The 2012 XMM-Newton observation of Sgr B2 clearly revealed a significantly increased $6.4 \mathrm{keV}$ flux, twice that of 2004 and higher than the central Sgr B2 area. The $6.4 \mathrm{keV}$ line emission contours shown in Figure 1 also show that the brightest subregions within G0.66 -0.13 were brighter than the Sgr B2 core at $6.4 \mathrm{keV}$ in 2012. The G0.66-0.13 Fe K $\alpha$ emission experienced an increase prior to 2012 and a fast decay from 2012 to 2013. However, the $6.4 \mathrm{keV}$ line flux sharply decreases and results in a nondetection by NuSTAR in 2013, giving an upper limit $(90 \%$ confidence level) to the $6.4 \mathrm{keV}$ line flux of $5 \times 10^{-6}$ photons $\mathrm{cm}^{-2} \mathrm{~s}^{-1}$. The $6.4 \mathrm{keV}$ line flux in 2013 is less than $50 \%$ of the value measured by XMM-Newton in 2012. It requires a short lifetime of $\sim 1 \mathrm{yr}$, which roughly matches the light-crossing time of the two bright cores within G0.66 $-0.13(2-3 \mathrm{yr})$.

\section{BROADBAND 2013 SPECTRA OF CENTRAL SGR B2 AND G0.66-0.13}

\subsection{Central $90^{\prime \prime}$ Radius Region of Sgr B2}

We extracted a NUSTAR spectrum from the central $90^{\prime \prime}$ radius region of Sgr B2 (R.A. $=17^{\mathrm{h}} 47^{\mathrm{m}} 20^{\mathrm{s}} .4$, decl. $=-28^{\circ} 23^{\prime} 07^{\prime \prime}$, 0 , J2000). The background subtraction method leaves mainly the molecular cloud emission and previously detected X-ray point sources. Thus, we first checked the flux contribution from known point sources in our source and background regions. The background regions we use do not contain any point sources above the Chandra detection threshold. In the source region, there are four point sources detected by Chandra within 90" of Sgr B2: CXOUGC J174723.0-282231, CXOUGC J174720.1-282305, CXOUGC J174718.2-282348, and CXOUGC J174713.7-282337. Takagi et al. (2002) suggested that the spectrum of these point sources can be best fit with a thermal plasma model of $\sim 5 \mathrm{keV}$, and proposed that they could be young stellar objects (YSOs). We checked each of the four sources using the stacked spectrum made from all archived Chandra data between 1999 September 21 and 2012 October 31 available for Sgr B2. Their summed observed flux corresponds to $\sim 7 \%$ of the total observed flux of the central $90^{\prime \prime}$ region below $10 \mathrm{keV}$. By extrapolating their spectra into a higher-energy band, we estimate that their flux contribution above $10 \mathrm{keV}$ is only $\sim 4 \%$. Therefore, the flux contribution from point sources is not significant for our study. Their spectra can be best fit with a collisionally ionized plasma model apec with a temperature of $\sim 3 \mathrm{keV}$, consistent with Takagi et al. (2002) within error bars. We thus used this model to represent unresolved X-ray point sources and any possible residual GC diffuse X-ray background within the source region.

\subsubsection{Ad Hoc XRN Model}

To examine the XRN scenario, we first applied a popular XRN model, with the wabs absorption model for a direct comparison with previous work. The model is composed of a power law and two Gaussians representing the $\mathrm{Fe} \mathrm{K} \alpha$ emission line at $6.40 \mathrm{keV}$ and the $\mathrm{K} \beta$ line at $7.06 \mathrm{keV}$, modified by intrinsic absorption column density. All the model components are subject to the foreground absorption, resulting in the XSPEC model wabs*(apec+wabs*powerlaw+gauss tgauss). We fixed the line energies of the two Gaussians to be $6.40 \mathrm{keV}$ (best-fit centroid energy of the $\mathrm{Fe} \mathrm{K} \alpha$ line of $\sim 6.44 \mathrm{keV}$ ) and $7.06 \mathrm{keV}$, with the normalization ratio of $\mathrm{K} \beta / \mathrm{K} \alpha$ set to $15 \%$ (Murakami et al. 2001).

The $3-79 \mathrm{keV}$ spectrum is well fit with this simple model $\left(\chi_{\nu}^{2}=0.97\right.$ for dof $\left.=91\right)$. The best-fit model parameters are listed in Table 2. The temperature of the thermal component is $2 \pm 1 \mathrm{keV}$ with an unabsorbed $2-10 \mathrm{keV}$ flux of $F_{2-10 \mathrm{keV}}=(6 \pm 4) \times 10^{-13} \mathrm{erg} \mathrm{cm}^{-2} \mathrm{~s}^{-1}$, consistent with the spectra of the point sources within $90^{\prime \prime}$ of Sgr B2. The $6.4 \mathrm{keV} \mathrm{Fe} \mathrm{K} \alpha$ line has an observed flux of $F_{6.4 \mathrm{keV}}=$ $(8.3 \pm 2.1) \times 10^{-6}$ photons $\mathrm{cm}^{-2} \mathrm{~s}^{-1}$. We calculated its $\mathrm{EW}$ based on the power law as the only continuum component, resulting in $\mathrm{EW}=1.2_{-0.3}^{+0.7} \mathrm{keV}$. The best-fit power-law photon index is $\Gamma=1.9 \pm 0.5$, consistent with previous measurements of $\Gamma \approx 2$ (Terrier et al. 2010). The observed $10-40 \mathrm{keV}$ flux is $F_{10-40 \mathrm{keV}}=(1.9 \pm 0.2) \times 10^{-12} \mathrm{erg} \mathrm{cm}^{-2} \mathrm{~s}^{-1}$. The intrinsic absorption column density is found to be $N_{\mathrm{H}}(i)=$ $(5.0 \pm 1.3) \times 10^{23} \mathrm{~cm}^{-2}$, on the lower end of, but still consistent with, the previous result of $(6.8 \pm 0.5) \times$ $10^{23} \mathrm{~cm}^{-2}$ derived using the combined 2003 XMM-Newton and 2003-2004 INTEGRAL/IBIS data (Terrier et al. 2010). All components are subject to a foreground interstellar column density of $N_{\mathrm{H}}(f)=(1.1 \pm 0.4) \times 10^{23} \mathrm{~cm}^{-2}$. The foreground column density value is consistent with our analysis of accumulated 2001-2012 XMM-Newton data of the inner $90^{\prime \prime}$ of Sgr B2, which gives $N_{\mathrm{H}}(i)=(1.0 \pm 0.2) \times 10^{23} \mathrm{~cm}^{-2}$. 
Table 2

Spectral Analysis of Central 90" of Sgr B2 with NuSTAR Data

\begin{tabular}{|c|c|c|c|c|c|}
\hline Parameter & Unit & Power-law Model $^{\mathrm{a}}$ & MyTorus Model $^{\mathrm{b}}$ & LECRe Mode $I^{c}$ & LECRp Model $^{\mathrm{d}}$ \\
\hline$\overline{N_{\mathrm{H}}(f)}$ & $10^{23} \mathrm{~cm}^{-2}$ & $1.1 \pm 0.4$ & $1.2 \pm 0.1$ & $1.3 \pm 0.5$ & $1.2 \pm 0.6$ \\
\hline$N_{\mathrm{H}}(i)$ & $10^{23} \mathrm{~cm}^{-2}$ & $5.0 \pm 1.3$ & $10.1 \pm 4.1$ & $5.5 \pm 1.3$ & $6.5_{-2.6}^{+3.9}$ \\
\hline$k T$ & $\mathrm{keV}$ & $2.1_{-0.9}^{+1.1}$ & $3.5_{-1.4}^{+2.5}$ & $3.4_{-1.2}^{+2.3}$ & $2.6_{-0.7}^{+1.4}$ \\
\hline$F_{\text {apec }}(2-10 \mathrm{keV})$ & $\mathrm{erg} \mathrm{cm}^{-2} \mathrm{~s}^{-1}$ & $6_{-4}^{+3} \times 10^{-13}$ & $6_{-3}^{+2} \times 10^{-13}$ & $7_{-3}^{+4} \times 10^{-13}$ & $6 \pm 3 \times 10^{-13}$ \\
\hline$F_{6.4 \mathrm{keV}}$ & $10^{-6}$ photons $\mathrm{cm}^{-2} \mathrm{~s}^{-1}$ & $8.3 \pm 2.1$ & $\ldots$ & $\ldots$ & $\ldots$ \\
\hline$F_{\text {p.l. }}(10-40 \mathrm{keV})$ & $\operatorname{erg~cm}{ }^{-2} \mathrm{~s}^{-1}$ & $(1.9 \pm 0.2) \times 10^{-12}$ & $\ldots$ & $\ldots$ & $\ldots$ \\
\hline$\Gamma_{\mathrm{MT}}$ & $\cdots$ & $\ldots$ & $2.2 \pm 0.4$ & $\ldots$ & $\cdots$ \\
\hline$N_{\mathrm{MT}}$ & $10^{-2}$ photons $\mathrm{cm}^{-2} \mathrm{~s}^{-1}$ & $\ldots$ & $1.8_{-1.1}^{+0.7}$ & & \\
\hline$\Lambda$ & $\mathrm{H}$-atoms $\mathrm{cm}^{-2}$ & $\ldots$ & $\ldots$ & $5 \times 10^{24}($ fixed $)$ & $5 \times 10^{24}($ fixed $)$ \\
\hline$s$ & $\cdots$ & $\ldots$ & $\ldots$ & $2.8_{-0.5}^{+0.4}$ & $1.9_{-0.7}^{+0.8}$ \\
\hline$E_{\min }$ & $\mathrm{keV}$ & $\ldots$ & $\ldots$ & $3_{-\ldots}^{+47}$ & $10^{4}$ (fixed) \\
\hline
\end{tabular}

Notes. The goodness of fit is evaluated by $\chi_{\nu}^{2}$ and the number of degrees of freedom given in parentheses. The errors are $90 \%$ confidence.

${ }^{a}$ Power-law model: wabs* (apec+wabs*pow+gauss+gauss). Power-law model is characterized by foreground interstellar absorption column density $N_{\mathrm{H}}(f)$, temperature $k T$ and $2-10 \mathrm{keV}$ flux $F_{\text {apec }}(2-10 \mathrm{keV})$ of apec, intrinsic absorption column density $N_{\mathrm{H}}(i)$, photon index $\Gamma_{\text {p.l. }}$ and $10-40 \mathrm{keV}$ observed flux $F_{\text {p.l. }}(10-40 \mathrm{keV})$ of power law, equivalent width EW, and observed flux $F_{6.4 \mathrm{keV}}$ of the $6.4 \mathrm{keV}$ iron line.

b MYTorus model: wabs* (apec+MYTorus). The MYTorus model is characterized by $N_{\mathrm{H}}(f), k T$, and $F_{\text {apec }}(2-10 \mathrm{keV})$ of apec, the intrinsic absorption column density $N_{\mathrm{H}}(i)$, photon index $\Gamma_{\mathrm{MT}}$ of the illuminating power-law spectrum, and the power-law normalization $N_{\mathrm{MT}}$, while the inclination angle is fixed to $\theta_{\mathrm{obs}}=0^{\circ}$.

${ }^{c}$ LECRe model: wabs* (apec+wabs*LECRe). The LECRe model is characterized by $N_{\mathrm{H}}(f), N_{\mathrm{H}}(i), k T$, and $F_{\text {apec }}(2-10 \mathrm{keV})$ of apec, the power-law index $s$ of the accelerated particle source spectrum varying from 1.5 to 5 , the path length $\lambda$ of the CRs in the nonthermal X-ray production region varying from $10^{21}$ to $10^{26} \mathrm{H}$-atoms $\mathrm{cm}^{-2}$, minimum energy $E_{\min }$, and the model normalization $N_{\mathrm{LECR}}$ for LECRe. $N_{\mathrm{LECR}}$ provides the power injected in the interaction region by primary $\mathrm{CR}$ electrons or protons of kinetic energies between $E_{\min }$ and $E_{\max }=1 \mathrm{GeV}$.

d LECRp model: wabs* (apec+wabs*LECRp). The LECRp model is characterized by $N_{\mathrm{H}}(f), N_{\mathrm{H}}(i), k T$, and $F_{\text {apec }}(2-10 \mathrm{keV})$ of apec, $s$ ranging from 1 to $5, \lambda$ ranging from $10^{21}$ to $10^{26} \mathrm{H}$-atoms $\mathrm{cm}^{-2}, E_{\min }$, and the model $N_{\mathrm{LECR}}$ for LECRp.

Although this ad hoc model can fit well to the data, it is not self-consistent, with the continuum emission and fluorescence lines decoupled. A power law can only measure the spectral slope of the observed scattered continuum, but not the illuminating source spectrum. The model is valid for measuring the illuminating source spectrum only when a molecular cloud is optically thin $\left(N_{\mathrm{H}} \ll 10^{24} \mathrm{~cm}^{-2}\right)$ and the Compton scattering is negligible. Interpretation of the resultant best-fit value for the intrinsic column density $N_{\mathrm{H}}(i)$ calls for caution. As this ad hoc model does not take cloud geometry into account, the $N_{\mathrm{H}}(i)$ measured by this model represents a characteristic column density of the cloud, while in reality illuminating X-ray photons are absorbed and scattered in various locations of the cloud.

\subsubsection{Self-consistent XRN Model MYTorus}

In the XRN scenario, to consistently measure the illuminating X-ray spectrum and to properly determine the intrinsic column density, a self-consistent XRN model based on Monte Carlo simulations is required. Murakami et al. (2001), Revnivtsev et al. (2004), Terrier et al. (2010), and Odaka et al. (2011) have applied Monte-Carlo-based XRN models to Sgr B2 data in order to study its morphology and spectrum. The MYTorus model is the only XRN model available in $\mathrm{XSPEC}$ to self-consistently measure the illuminating source spectrum and the intrinsic column density (Murphy \& Yaqoob 2009). MYTorus was originally developed for Compton-thick AGNs assuming a toroidal reflector with neutral materials and uniform density. The default $\mathrm{Fe}$ abundance in the MYTorus model is one solar and does not allow variation. There are three components offered in the model: the transmitted continuum (MYTZ), the scattered continuum (MYTS), and the iron fluorescence lines (MYTL). In the case of GCMCs, the observed spectrum only contains the last two components, because we are seeing only the reflected X-ray photons off the cloud. We thus use the combination of MYTS and MYTL. Both components depend on the intrinsic equatorial hydrogen column density $N_{\mathrm{H}}(i)$, the illuminating source spectrum photon index $\Gamma$, the inclination angle $\theta_{\mathrm{obs}}$ between the line of sight and the torus symmetry axis, and the model normalization $N_{\mathrm{MT}}$. To self-consistently measure the illuminating source spectrum, all the parameters are linked between MYTS and MYTL as a coupled mode where the same incident X-ray spectrum is input into both components. We select a termination energy of the incident power law as $500 \mathrm{keV}$. As the best-fit energy centroid for the $\mathrm{Fe} \mathrm{K} \alpha$ line is $\sim 6.44 \mathrm{keV}$, we select an energy offset of $+40 \mathrm{eV}$ for the MYTL component to allow freedom for the centroid energy. The resultant model is wabs* (apec+MYTS +MYTL), where the two MYTorus components are implemented as table models in Xspec: atable\{mytorus_scatteredH500_v00.fits $\}+$ atable\{mytl_v000010 pEp040H500_v00.fits\}.

The coupled mode of the MYTorus model can be applied to the GCMC X-ray reflection spectra with some restrictions, by treating a quasi-spherical cloud as part of a virtual torus and rescaling incident X-ray flux properly (see Figure 6 below for geometry). Owing to the toroidal geometry and the uniform density profile MYTorus assumes, for GCMCs we restrict the applicability of the model to the inclination angle range of 

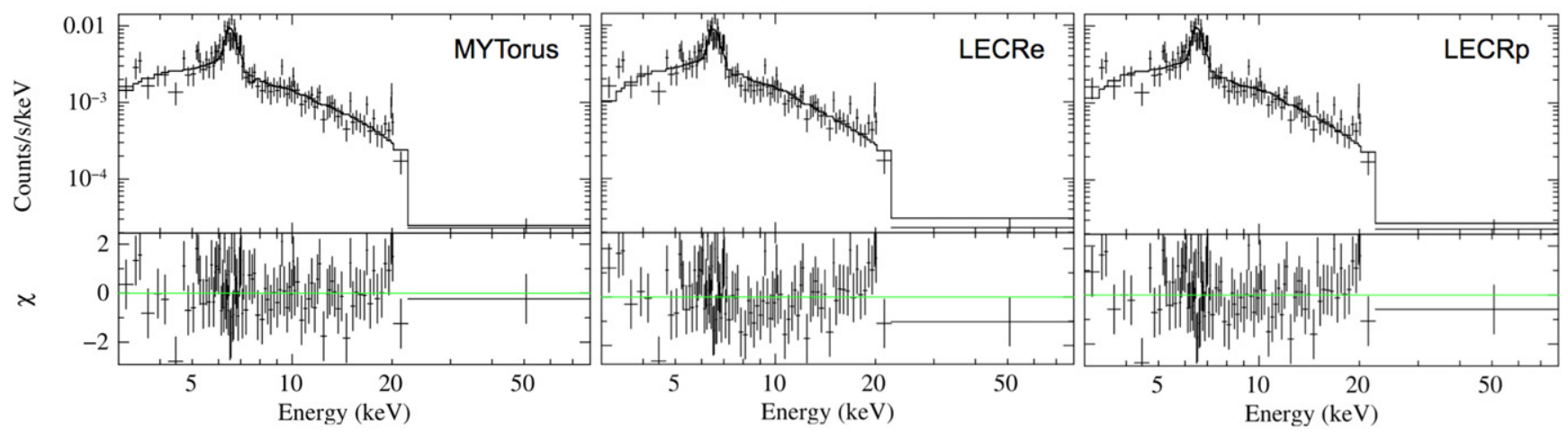

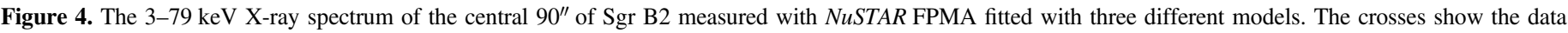

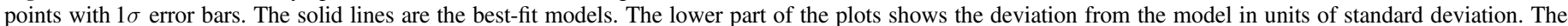

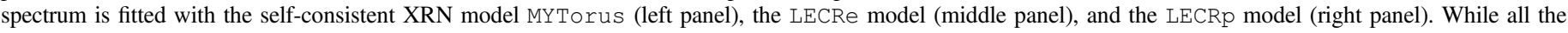

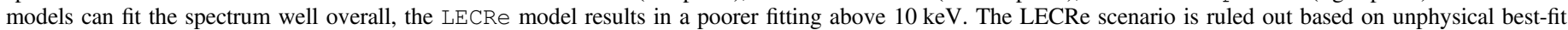
model parameters.

$\theta_{\text {obs }} \lesssim 60^{\circ}$ and the equatorial column density range of $N_{\mathrm{H}} \lesssim 10^{24} \mathrm{~cm}^{-2}$, in order to be insensitive to the torus geometry (see Appendix for more details). When $N_{\mathrm{H}}(i)$ reaches $\sim 10^{24} \mathrm{~cm}^{-2}$, we derive the systematic error based on the MYTorus model to be $25 \%$ for $N_{\mathrm{H}}, 3 \%$ for $\Gamma$, and $10 \%$ for the model normalization (Mori et al. 2015). We note that the $N_{\mathrm{H}}(i)$ measured by the MYTorus model is an averaged value over the torus.

This self-consistent model can fit to the Sgr B2 spectrum well, yielding $\chi_{\nu}^{2}=1.09$ for dof $=92$ (left panel of Figure 4). Both the best-fit temperature and the flux of the apec component are consistent with the fitting results of the ad hoc power-law model (Table 2). The foreground column density is $N_{\mathrm{H}}(f)=(1.2 \pm 0.1) \times 10^{23} \mathrm{~cm}^{-2}$, consistent with that derived from the ad hoc model and better constrained. However, the intrinsic equatorial column density is $N_{\mathrm{H}}(i)=\left(1.01 \pm 0.16_{\text {stat }} \pm 0.25_{\text {sys }}\right) \times 10^{24} \mathrm{~cm}^{-2}$, which is twice the $N_{\mathrm{H}}(i)$ value measured with the ad hoc model. The $N_{\mathrm{H}}(i)$ value derived with the two models cannot be directly compared owing to the lack of geometry definition for $N_{\mathrm{H}}(i)$ in the ad hoc model. $N_{\mathrm{H}}(i)$ in the MYTorus model corresponds to the minor diameter of the torus (or diameter of the quasispherical cloud), while $N_{\mathrm{H}}(i)$ in the ad hoc model roughly corresponds to the cloud radius as it "measures" an averaged effect, which might explain the difference by a factor of 2 (see the Appendix). The best-fit $N_{\mathrm{H}}(i)$ value derived by the MYTorus model is consistent with the molecular line emission measurements, which indicate an $\mathrm{H}_{2}$ column density of $N_{\mathrm{H}} \sim 10^{24} \mathrm{~cm}^{-2}$ (Lis \& Goldsmith 1990). For the central $90^{\prime \prime}$ of Sgr B2, the cloud radial optical depth due to Thomson scattering derived by the MYTorus model is $\tau_{\mathrm{T}}=0.67 \pm$ 0.27. As a result, the central $90^{\prime \prime}$ of $\mathrm{Sgr} \mathrm{B} 2$ is marginally optically thin. The illuminating source spectrum photon index is constrained to be $\Gamma=2.2 \pm 0.4$, with the systematic error being negligible, compatible with the observed spectrum slope of $\Gamma=1.9 \pm 0.5$ measured by the power-law model, which does not consider Compton scattering. With the hard upper limit on the inclination angle $\theta_{\mathrm{obs}}=60^{\circ}$ chosen for this study, the MYTorus model is not sensitive to the parameter $\theta_{\text {obs. }}$. Therefore, this parameter could not be constrained by our work.

Though with limitations, MYTorus is currently the best selfconsistent XRN model available. The systematic errors could be underestimated since they are estimated solely based on the MYTorus model (see the Appendix). A modified XRN model based on MYTOrus with a spherical reflector and variable $\mathrm{Fe}$ abundance is under development for the specific case of GCMCs, which will remove the uncertainties induced by the toroidal reflector and the fixed Fe abundance (T. Yaqoob 2015, private communication). The more sophisticated XRN model will also implement the specific density profile of Sgr B2 to remove uncertainties induced by nonuniform density and better constrain $N_{\mathrm{H}}(i)$ (M. Walls et al. 2015, in preparation).

\subsubsection{Self-consistent Cosmic-ray Electron Model LECRe}

To determine whether $\mathrm{CR}$ electrons can be a major contributor to the remaining Sgr B2 X-ray emission in 2013, we tested the LECRe model (Tatischeff et al. 2012). The LECRe model has four free parameters: the power-law index of the accelerated particle source spectrum, $s$, the path length of $\mathrm{CRs}$ in the nonthermal X-ray production region, $\Lambda$, the minimum electron energy, $E_{\mathrm{min}}$, and the model normalization, $N_{\text {LECR }}$, which provides the power injected in the interaction region by primary $\mathrm{CR}$ electrons of kinetic energies between $E_{\min }$ and $E_{\max }=1 \mathrm{GeV}: d W / d t=4 \pi D^{2} N_{\mathrm{LECR}} \mathrm{erg} \mathrm{s}^{-1}$, where $D$ is the source distance. Since the fit cannot constrain the path length of LECRe, we fix it to $\Lambda=5 \times 10^{24} \mathrm{H}$-atoms $\mathrm{cm}^{-2}$ as was used in Tatischeff et al. (2012). As shown in the middle panel of Figure 4, it results in an acceptable fit to the data with $\chi_{\nu}^{2}=1.18$ for dof $=92$ (Table 2). The best-fit electron spectral index is $s=2.8 \pm 0.5$, with an intrinsic column density of $N_{\mathrm{H}}=(5.5 \pm 1.3) \times 10^{23} \mathrm{~cm}^{-2}$. Previous analysis with XMM-Newton and INTEGRAL/IBIS data found that $s \sim 1.5$, but with an unconstrained error bar (Terrier et al. 2010). The best-fit minimum electron energy is as low as $3 \mathrm{keV}$, with an upper bound of $50 \mathrm{keV}$. Electrons with such low energy cannot leave their acceleration site or penetrate the cloud. The best-fit molecular cloud metallicity is $Z / Z_{\odot}=4.0_{-0.6}^{+2.0}$, higher than the $Z / Z_{\odot}=3.1 \pm 0.2$ derived by Terrier et al. (2010). Such a metallicity value is too high compared to current measurements of the GC interstellar medium metallicity, which ranges from slightly higher than solar to twice solar. We also explored the best-fit $s$ values with different $N_{\mathrm{H}}$ values and found that $s=2.1-3.2$, corresponding to $N_{\mathrm{H}}=(1-10) \times 10^{23} \mathrm{~cm}^{-2}$. In these parameter ranges, the required metallicity is always $Z / Z_{\odot}>3$, and the minimum electron energy is $E_{\min }<100 \mathrm{keV}$. 
Furthermore, compared to the XRN models, the LECRe model results in a poorer fit above $10 \mathrm{keV}$ as it cannot fit to the spectrum curvature equally well. The high-energy part of the spectrum thus provides a more excluding constraint on the LECRe model, which does not depend on the high metallicity required by the significant $\mathrm{Fe} \mathrm{K} \alpha$ line. We therefore confirm that the unphysical best-fit model parameters make the LECRe scenario unlikely to be a major process to account for the Sgr B2 X-ray emission in 2013. If there is an underlying contribution from CR electrons, it has to be significantly lower than the current level.

\subsubsection{Self-consistent Cosmic-ray Proton Model LECRp}

While CR electrons can be safely excluded as a major contributor to the 2013 Sgr B2 X-ray emission, low-energy CR proton/ion bombardment could be a major process if the Sgr B2 X-ray emission obtained in 2013 has already reached the constant background level. The LECRp model has the same model parameters as the LECRe model, with four free parameters $s$, $\Lambda, E_{\min }$, and $N_{\mathrm{LECR}}$. As the path length cannot be constrained by the fit, we fix it to $\Lambda=5 \times 10^{24} \mathrm{H}$-atoms $\mathrm{cm}^{-2}$, a typical value for nonrelativistic particles propagating in massive molecular clouds of the GC environment (Tatischeff et al. 2012). The right panel in Figure 4 shows that it results in an overall good fit with $\chi_{\nu}^{2}=1.17$ for dof $=92$ (Table 2). The best-fit Fe abundance is $Z / Z_{\odot}=2.5_{-1.0}^{+1.5}$, which is consistent with the GC metallicity. The CR proton spectral index is $s=1.9_{-0.7}^{+0.8}$. This agrees with the CR proton spectral index derived with the LECRp model for the arches region using the XMM-Newton spectrum $\left(s=1.9_{-0.6}^{+0.5}\right.$, Tatischeff et al. 2012) or the NuSTAR spectrum $(s=1.7 \pm 0.6$, Krivonos et al. 2014). CR iron bombardment was proposed by these authors to explain the X-ray emission from the Arches. As discussed in Tatischeff et al. (2012), with such a relatively hard $\mathrm{CR}$ spectrum, the $\mathrm{X}$-ray emission produced by CR protons depends weakly on the minimum ion energy $E_{\min }$. Therefore, we fix it to $E_{\min }=10 \mathrm{MeV}$ nucleon $^{-1}$. The total power required by $\mathrm{CR}$ protons in the cloud can be derived from the best-fit model normalization $N_{\mathrm{LECR}}=$ $(1.4 \pm 0.4) \times 10^{-7} \mathrm{erg} \mathrm{cm}^{-2} \mathrm{~s}^{-1}$. The power injected by primary protons of energies between $E_{\min }=10 \mathrm{MeV}$ and $E_{\max }=1 \mathrm{GeV} \quad$ is $\quad d W / d t=(1.0 \pm 0.3) \times 10^{39} \mathrm{erg} \mathrm{s}^{-1}$ for $D=7.9 \mathrm{keV}$. However, the power injected into the cloud by the $\mathrm{CR}$ protons depends on the minimum energy $E_{\min }$. For $E_{\min }=1 \mathrm{MeV}$ and $E_{\min }=100 \mathrm{MeV}$, the best-fit model normalizations are $N_{\mathrm{LECR}}=2.0_{-0.5}^{+1.1} \times 10^{-7} \mathrm{erg} \mathrm{cm}^{-2} \mathrm{~s}^{-1}$ and $N_{\mathrm{LECR}}=0.7_{-0.1}^{+0.3} \times 10^{-7} \mathrm{erg} \mathrm{cm}^{-2} \mathrm{~s}^{-1}$, respectively. The corresponding power injected by $\mathrm{CR}$ protons is in the range of $d W / d t=(0.4-2.3) \times 10^{39} \mathrm{erg} \mathrm{s}^{-1}$. Therefore, the LECRp scenario could explain the current-level Sgr B2 X-ray emission and can only be excluded by further variability.

\subsection{Main Compact Cores Sgr B2(M) and Sgr B2(N)}

By studying the $250 \mu \mathrm{m}$ continuum emission obtained with Herschel, Etxaluze et al. (2013) show that the local optical depth at Sgr B2(M) and Sgr B2(N) $\left(\tau_{250} \mu \mathrm{m} \sim 1\right)$ is higher than that of the surrounding region within $90^{\prime \prime}$ roughly by a factor of $2-5$. The local column density of $\operatorname{Sgr} \mathrm{B} 2(\mathrm{M})$ is therefore $N_{\mathrm{H}} \sim 1.8 \times 10^{24} \mathrm{~cm}^{-2}$ based on the conversion relationship $\tau_{250 \mu \mathrm{m}} / N_{\mathrm{H}}=5 \times 10^{-25} \mathrm{~cm}^{2}$ for the Sagittarius region (Bernard et al. 2010). The molecular line emission measurements also revealed that the Sgr B2(M) column density is
$N_{\mathrm{H}} \sim 2 \times 10^{24} \mathrm{~cm}^{-2}$ (Lis \& Goldsmith 1990). We extracted a spectrum from a $20^{\prime \prime}$ radius region (Figure 2) centered on Sgr B2(M) to measure the local optical depth independently using the X-ray data. The Sgr B2(M) spectrum is fit with the MYTorusmodel using the same model settings for the central $90^{\prime \prime}$ region. The model yields a good fit with $\chi_{\nu}^{2}=1.06$ for dof $=39$. While all the other key parameters are consistent with those derived from the central $90^{\prime \prime}$ region, the intrinsic equatorial column density is $N_{\mathrm{H}}(i)=\left(2.0_{-1.0_{\mathrm{stat}}+0.5_{\mathrm{sys}}}^{+3.2_{\mathrm{sys}}+0.5_{\mathrm{sy}}} \times 10^{24} \mathrm{~cm}^{-2}\right.$, twice that of the whole $90^{\prime \prime}$ region. The LECRp model can also fit well to the Sgr B2(M) spectrum $\left(\chi_{\nu}^{2}=1.02\right.$ for dof $\left.=39\right)$, giving consistent $N_{\mathrm{H}}(i)$ value. It agrees well with the local column density value derived from molecular line emission and the $250 \mu \mathrm{m}$ continuum emission. The corresponding radial optical depth in the direction of $\operatorname{Sgr} \mathrm{B} 2(\mathrm{M})$ is $\tau_{\mathrm{T}}=1.3_{-1.0}^{+2.5}$. The NuSTAR observations confirm that the Sgr B2(M) region is not optically thin, though with large error bars. The $10-40 \mathrm{keV}$ flux of Sgr B2(M) and Sgr B2(N) falling into the central $90^{\prime \prime}$ region is $F_{10-40 \mathrm{keV}}=(6.1 \pm 0.2) \times 10^{-13} \mathrm{erg} \mathrm{cm}^{-2} \mathrm{~s}^{-1}$, contributing to about one-third of the total flux of the central $90^{\prime \prime}$. The surrounding regions within the $90^{\prime \prime}$ radius region contribute to the remaining two-thirds of the total flux. The second core, Sgr B2(N), does not have sufficient statistics to perform a similar analysis.

\subsection{The New Feature G0.66-0.13}

Since neither of the focal plane modules are severely contaminated by stray light, we were able to use the combined FPMA and FPMB NuSTARdata for G0.66-0.13. Furthermore, the G0.66-0.13 region does not contain sources registered in the Chandra point-source catalog (Muno et al. 2009). The back-

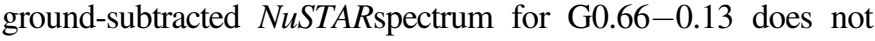
show any significant line features. To compare with the significant $\mathrm{Fe} \mathrm{K} \alpha$ lines detected by XMM-Newton in 2012, we fit the NuSTAR 2013 spectrum together with the XMM-Newton 2012 spectrum. Since the sharply decreasing Fe K $\alpha$ emission cannot be explained by the LECRp scenario, we examine the G0.66-0.13 spectrum only with the MYTorus model. Because of different background subtraction methods, the thermal component in the $X M M$-Newton data represents the GC diffuse emission, while the thermal component in the NuSTARdata represents undetected point-source and residual diffuse emission. Therefore, we use the model wabs* (apec+MYTS+MYTL) for the joint fitting, with all the model parameters linked except for $k T$ and the model normalizations of apec,MYTS, and MYTL. The model gives a good fit $\left(\chi_{\nu}^{2}=1.18\right.$ for dof $\left.=55\right)$, with a best-fit photon index of $\Gamma=1.4 \pm 0.5$, a foreground column density of $N_{\mathrm{H}}(f)=\left(8.2_{-4.5}^{+4.3}\right) \times 10^{22} \mathrm{~cm}^{-2}$, and an intrinsic equatorial column density of $N_{\mathrm{H}}(i)=3.0_{-1.9}^{+3.8} \times 10^{23} \mathrm{~cm}^{-2}$ (Figure 5). The intrinsic density $N_{\mathrm{H}}(i)$ is lower than that of the central $90^{\prime \prime}$ radius region, indicating that G0.66-0.13 is optically thin. However, in case that G0.66-0.13 is partially illuminated, the measured $N_{\mathrm{H}}(i)$, which is the illuminated column density, would be smaller than the cloud intrinsic column density. The $10-40 \mathrm{keV}$ flux for the NuSTAR 2013 data is $F_{10-40 \mathrm{keV}}=(9.0 \pm 1.2) \times 10^{-13} \mathrm{erg} \mathrm{cm}^{-2} \mathrm{~s}^{-1}$ in $10-40 \mathrm{keV}$. The observed $6.4 \mathrm{keV}$ flux in 2012 measured by XMM-Newton is $F_{6.4 \mathrm{keV}}=(1.5 \pm 0.2) \times 10^{-5}$ photons $\mathrm{cm}^{-2} \mathrm{~s}^{-1}$, while the $2013 \mathrm{NUSTAR}$ spectrum does not require a $6.4 \mathrm{keV}$ line, with a $6.4 \mathrm{keV}$ flux upper limit of $5 \times 10^{-6}$ photons $\mathrm{cm}^{-2} \mathrm{~s}^{-1}(90 \%$ confidence level). The $8-12 \mathrm{keV}$ nonthermal continuum 


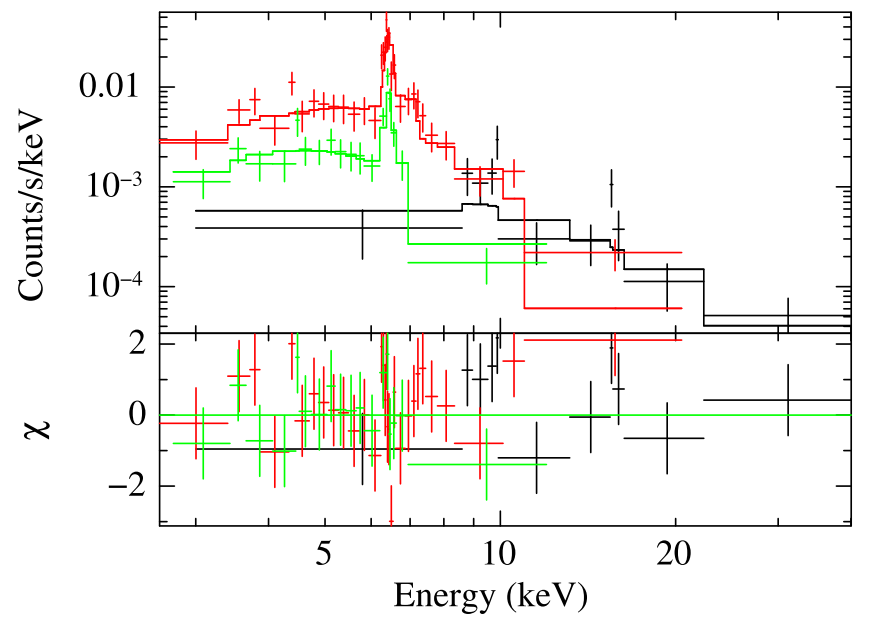

Figure 5. 2013 NUSTAR (black) and 2012 XMM-Newton (red for PN and green for MOS) spectra of G0.66-0.13 jointly fit to the XRN model wabs* (apec + MYTS+MYTL). The crosses show the data points with $1 \sigma$ error bars, and the solid lines show the best model. The lower panel shows the deviation from the model in units of standard deviation. The $6.4 \mathrm{keV}$ line was significantly detected by XMM-Newton in 2012, but not detected by NuSTAR in 2013. It suggests a fast decay of fluorescence emission within $1 \mathrm{yr}$.

emission measured by NuSTAR in 2013 dropped to $\sim 50 \%$ of that measured by XMM-Newton in 2012. Both the fluorescence emission and the hard X-ray emission show fast variability (or short lifetime), which could be due to a partial illumination of G0.66-0.13.

\section{DISCUSSION}

\subsection{X-Ray Reflection of Past Sgr A* Outbursts}

The broadband spectrum obtained by NuSTAR supports the XRN scenario where Sgr B2 reflects incoming X-rays from an illuminating source, which is most likely a past X-ray outburst from Sgr $\mathrm{A}^{\star}$. The illuminating source photon index measured with the broadband NuSTAR spectrum in 2013 using the MyTorus model is constrained to $\Gamma=2.2 \pm 0.4$, compatible with the illuminating source photon index of $\Gamma=2.0 \pm 0.2$ derived with combined XMM-Newton and INTEGRAL/IBIS spectra obtained in 2003-2004 when Sgr B2 was much brighter (Terrier et al. 2010). The outburst spectrum is also compatible with, though at the extreme end of, the average AGN photon index of $1 \leqslant \Gamma \leqslant 2$, with cutoff energies between 30 and $300 \mathrm{keV}$ (Molina et al. 2013), which is also expected for the Sgr $A^{\star}$ spectrum if it became active.

The Sgr B2 cloud as a whole has been discussed as an optically thin cloud (e.g., Koyama et al. 1996; Sunyaev \& Churazov 1998; Revnivtsev et al. 2004; Terrier et al. 2010). In 2013, NuSTAR found that the hard X-ray emission is concentrated in the central $90^{\prime \prime}$ radius region of Sgr B2, but it is unknown whether the hard X-ray emission in 2003-2009 was similarly concentrated within $90^{\prime \prime}$ or more extended owing to the mild angular resolution of INTEGRAL/IBIS. While in the direction of the compact core Sgr B2(M) the optical depth is as high as $\tau_{\mathrm{T}} \gtrsim 1$, the majority of the central $90^{\prime \prime}$ radius region is optically thin with $\tau_{\mathrm{T}}<1$. Besides, the $10-40 \mathrm{keV}$ image shows that the X-ray continuum emission traces the local column density, also suggesting that the majority of the central $90^{\prime \prime}$ of Sgr B2 is optically thin. Therefore, although there are complicated substructures with higher local column densities, the central $90^{\prime \prime}$ can be considered as optically thin for a simplified calculation of Sgr $\mathrm{A}^{\star}$ outburst luminosity.

For an optically thin cloud, the luminosity of the primary source can be derived via two independent analytical methods: the $\mathrm{Fe} \mathrm{K} \alpha$ fluorescence process using the $6.4 \mathrm{keV}$ line flux we measured, and the Compton scattering process with the measured hard X-ray continuum. In most previous works, the primary source luminosity has been calculated via the fluorescence process, owing to good measurements of the $6.4 \mathrm{keV}$ line but poorly constrained continuum emission. The $6.4 \mathrm{keV}$ line emission flux for continuum radiation is expressed by Sunyaev \& Churazov (1998) as

$$
F_{6.4}=\phi \frac{\Omega}{4 \pi D^{2}} Z_{\mathrm{Fe}} \tau_{\mathrm{T}} I(8) \text { photons } \mathrm{cm}^{-2} \mathrm{~s}^{-1},
$$

where $\Omega$ is the cloud solid angle subtended to the illuminating source, $D$ is the distance to the observer, $Z_{\mathrm{Fe}}$ is the iron abundance relative to solar, $\tau_{\mathrm{T}}$ is optical depth due to Thomson scattering, and $I(8)$ is the illuminating source flux at $8 \mathrm{keV} . \phi$ is a factor of the order of unity, which depends on the source spectrum. Sunyaev \& Churazov (1998) assumed the source spectrum to be bremsstrahlung with a temperature from 5 to $150 \mathrm{keV}$, corresponding to $\phi \sim 1.0-1.3$. Now, with a better knowledge of the primary source spectrum, we know that it can be well described with a power law with $\Gamma \approx 2$. $\Gamma$ ranges from 1.8 to 2.6 , as measured with the MYTorus model, corresponding to $\phi \sim 1.1-1.3$. In the calculation hereafter we will adopt $\Gamma \approx 2$ and its corresponding $\phi=1.18$.

Using the Sunyaev \& Churazov (1998) approach, we express $I(8)$ with illuminating source luminosity at $8 \mathrm{keV}$ in the $8 \mathrm{keV}$ wide energy band as $L_{8}=I(8) \times 8 \times 8 \times 1.6 \times$ $10^{-9} \mathrm{erg} \mathrm{s}^{-1}$. For the central region of Sgr B2 we adopted $r=90^{\prime \prime}$, assuming that it was entirely illuminated, so the luminosity of the source required to produce the observed $6.4 \mathrm{keV}$ line is

$$
L_{8}=3 \times 10^{39}\left(\frac{F_{6.4}}{10^{-4}}\right)\left(\frac{0.1}{\tau_{\mathrm{T}}}\right)\left(\frac{d}{100 \mathrm{pc}}\right)^{2}\left(Z_{\mathrm{Fe}}\right)^{-1} \mathrm{erg} \mathrm{s}^{-1}
$$

where $d$ is the distance between the molecular cloud and the illuminating source. For a power-law spectrum with $\Gamma \approx 2, L_{8}$ corresponds to $30 \%$ of the source luminosity at $3-79 \mathrm{keV}$. While the $6.4 \mathrm{keV}$ line flux has been decreasing over the past decade, the maximum observed $\mathrm{Fe} \mathrm{K} \alpha$ flux should be used to calculate the peak primary source luminosity. As the XRN model we use does not measure the iron abundance of Sgr B2, we used the most recently measured value $Z_{\mathrm{Fe}}=1.3 \pm 0.1$ (Terrier et al. 2010). With the maximum central 90" Sgr B2 flux $F_{6.4}=(4.3 \pm 0.6) \times 10^{-5}$ photons $\mathrm{cm}^{-2} \mathrm{~s}^{-1}$ obtained in 2001 and averaged optical depth $\tau_{\mathrm{T}}=0.67 \pm 0.27$ measured with $N U S T A R$, the resultant primary source luminosity is $L_{8}=(1.5 \pm 0.7) \times 10^{38}(\mathrm{~d} / 100 \mathrm{pc})^{2} \mathrm{erg} \mathrm{s}^{-1}, \quad$ corresponding to a $3-79 \mathrm{keV}$ luminosity of $L_{3-79 \mathrm{keV}}=(5.0 \pm 2.3) \times$ $10^{38}\left(d / 100 \mathrm{pc}^{2} \mathrm{erg} \mathrm{s}^{-1}\right.$ or $1-100 \mathrm{keV}$ luminosity of $L_{1-100 \mathrm{keV}}=(0.7 \pm 0.3) \times 10^{39}(\mathrm{~d} / 100 \mathrm{pc})^{2} \mathrm{erg} \mathrm{s}^{-1}$. This is comparable to the $L_{1-100 \mathrm{keV}} \sim 1.1 \times 10^{39}(\mathrm{~d} / 100 \mathrm{pc})^{2} \mathrm{erg} \mathrm{s}^{-1}$ derived from XMM-Newton and INTEGRAL/IBIS data obtained in 2003-2004 (Terrier et al. 2010). The uncertainty of the primary source luminosity mainly comes from the measurement uncertainty of $\tau_{\mathrm{T}}$ and the distance between 
Sgr B2 and the primary source, $d$. The solid angle $\Omega$ could also be lower in the case of partial illumination, which would result in an underestimation of the primary source luminosity.

Since this was the first time the Sgr B2 $10-40 \mathrm{keV}$ continuum emission has been resolved, we can also derive the primary source continuum luminosity from the Comptonscattered continuum. As discussed in Capelli et al. (2012), the observed scattered continuum at an angle $\theta$ with respect to the incident radiation direction is

$$
S_{(E)}=\frac{\Omega}{D^{2}} \frac{3 \tau_{\mathrm{T}}}{8 \pi} \frac{\left(1+\cos ^{2} \theta\right)}{2} I(E) \text { photons } \mathrm{cm}^{-2} \mathrm{~s}^{-1} \mathrm{keV}^{-1}
$$

where $I(E)$ is the photon flux from the source in units of photons $\mathrm{s}^{-1} \mathrm{keV}^{-1}$. This relationship is based on Thomson scattering, which is a good approximation for incident photons with energies of a few $\mathrm{keV}$. However, when the photon energy is higher, electron quantum mechanical effects become important. As a result, we need to consider the Compton scattering process. Starting with the Compton scattering cross-section formula, we derived a correction factor from Thomson scattering to Compton scattering as $f_{\mathrm{TC}}=$ $\left(1+\frac{E}{511 \mathrm{keV}}(1-\cos \theta)\right)^{-4}$, assuming a power-law spectrum with $\Gamma \approx 2$. The correction holds as long as $\hbar \omega \ll m c^{2}$ for the electrons, which is true for the NUSTAR energy band of 3-79 keV.

Thus, for any X-ray photon energy, the Compton-scattered continuum can be expressed as

$$
\begin{aligned}
S(E)= & \frac{\Omega}{D^{2}} \frac{3 \tau_{\mathrm{T}}}{8 \pi} \frac{\left(1+\cos ^{2} \theta\right)}{2}\left(1+\frac{E}{511 \mathrm{keV}}(1-\cos \theta)\right)^{-4} \\
& \times I(E) \text { photons } \mathrm{cm}^{-2} \mathrm{~s}^{-1} \mathrm{keV}^{-1} .
\end{aligned}
$$

The observed $10-40 \mathrm{keV}$ scattered continuum flux is $F_{10-40}=$ $\int_{10 \mathrm{keV}}^{40 \mathrm{keV}} S(E) E d E \mathrm{erg} \mathrm{cm}^{-2} \mathrm{~s}^{-1}$. Thus, integrating both sides of Equation (4) gives us the relationship between $F_{10-40}$ and $L_{8}$. We keep the first-order correction term of the integrated form and neglect higher-order terms. As a result, the source luminosity in the $8 \mathrm{keV}$ wide band can be expressed with observed 10-40 keV Compton-scattered continuum measured at angle $\theta$ as

$$
\begin{aligned}
L_{8}= & 4 \times 10^{39}\left(\frac{F_{10-40}}{10^{-11} \mathrm{erg} \mathrm{cm}^{-2} \mathrm{~s}^{-1}}\right)\left(\frac{0.1}{\tau_{\mathrm{T}}}\right)\left(\frac{d}{100 \mathrm{pc}}\right)^{2} \\
& \times \frac{1}{1+\cos ^{2} \theta} \times(0.72+0.12(1-\cos \theta)) \mathrm{erg} \mathrm{s}^{-1} .
\end{aligned}
$$

We use the maximum value of the hard X-ray continuum flux in 2012 from the light curve obtained by XMM-Newton. In Section 5 we estimated the $10-40 \mathrm{keV}$ flux of the central $90^{\prime \prime}$ in 2001. The resultant source luminosity is $L_{8}=(3.6 \pm 1.8) \times 10^{38} f(\theta)(d / 100 \mathrm{pc})^{2} \mathrm{erg} \mathrm{s}^{-1}, \quad$ where $f(\theta)=\frac{1}{1+\cos ^{2} \theta}(0.72+0.12(1-\cos \theta)) \sim 0.36-0.84$ corresponding to $\theta \sim 0^{\circ}-180^{\circ}$. As the scattering angle range cannot be derived from the XRN model fitting in our study, we adopt $\cos \theta \sim 0.8$ based on very long baseline interferometry observation results (Reid et al. 2009) to derive a nominal value for $f(\theta)$. The error of $f(\theta)$ is calculated corresponding to the full allowed range of the scattering angle $\theta=0^{\circ}-180^{\circ}$, resulting in $f(\theta)=0.46_{-0.10}^{+0.38}$. Thus, $L_{8}=\left(1.7_{-0.9}^{+1.6}\right) \times$ $10^{38}(\mathrm{~d} / 100 \mathrm{pc})^{2} \mathrm{erg} \mathrm{s}^{-1}$, corresponding to a $3-79 \mathrm{keV}$ luminosity of $\quad L_{3-79 \mathrm{keV}}=\left(5.6_{-2.9}^{+5.2}\right) \times 10^{38}(\mathrm{~d} / 100 \mathrm{pc})^{2} \mathrm{erg} \mathrm{s}^{-1}$ or a $1-100 \mathrm{keV}$ luminosity of $L_{1-100 \mathrm{keV}}=\left(0.8_{-0.4}^{+0.7}\right) \times$ $10^{39}(d / 100 \mathrm{pc})^{2} \mathrm{erg} \mathrm{s}^{-1}$.

This result is consistent with $L_{8}$ calculated via the photoelectric absorption process. Indeed, comparing Equations (2) and (5), we have

$$
\frac{L_{8}(\mathrm{abs})}{L_{8}(\mathrm{scat})}=\frac{3\left(\frac{F_{10-40}}{10^{-11} \mathrm{erg} \mathrm{cm}^{-2} \mathrm{~s}^{-1}}\right)\left(Z_{\mathrm{Fe}}\right)^{-1}}{4\left(\frac{F_{6.4}}{10^{-4} \text { photons cm } \mathrm{cm}^{-2} \mathrm{~s}^{-1}}\right) f(\theta)}=0.8_{-0.6}^{+0.4},
$$

which is a factor of the order of unity. The primary source luminosity independently calculated via the Compton scattering is remarkably consistent with that calculated via photoelectric absorption. The consistency strongly supports the $\mathrm{X}$-ray reflection scenario. With Equation (4), the primary source luminosity in any energy band can be directly derived given the measurement of the molecular cloud flux in the same energy band. This continuum-flux-based method has also been applied to other GCMCs to test the XRN model and constrain the Sgr $A^{\star}$ outburst history (Mori et al. 2015).

The MYTorus model can also give the illuminating source spectrum, although it assumes fixed solar abundance for iron and that the observation angle cannot be constrained with the data. By rescaling the incoming X-ray flux by the solid angle ratio of the torus $(\Omega / 4 \pi=0.5)$ and Sgr B2 $\left(\Omega / 4 \pi=2.89 \times 10^{-4}\right)$, we derive the primary source luminosity to be $L_{8}=\left(1.4_{-0.1}^{+0.5}\right) \times 10^{38}(\mathrm{~d} / 100 \mathrm{pc})^{2} \mathrm{erg} \mathrm{s}^{-1}$ (see the Appendix for details), As the $2013 \mathrm{X}$-ray emission from Sgr B2 is $30 \%$ that of its maximum flux (Section 5), the primary source luminosity with the peak Sgr B2 flux is $L_{8}=\left(4.6_{-3.6}^{+1.6}\right) \times 10^{38}(d / 100 \mathrm{pc})^{2} \mathrm{erg} \mathrm{s}^{-1}, \quad$ or $\quad L_{3-79 \mathrm{keV}}=$ $\left(1.5_{-1.1}^{+0.5}\right) \times 10^{39}(d / 100 \mathrm{pc})^{2} \mathrm{erg} \mathrm{s}^{-1}$ with the $2001 \mathrm{Sgr}$ B2 $\mathrm{X}$-ray flux. With large error bars, the primary source luminosity derived by the MYTorus model overlaps with that derived with the analytical calculations, though the best-fit luminosity value is higher. For the other GCMCs, the MYTorus model also gives a primary source luminosity higher than that derived from the analytical calculation roughly by a factor of 2 (Mori et al. 2015).

We note that the analytical calculations above are valid for an optically thin $\left(\tau_{\mathrm{T}} \ll 1\right)$ cloud where the fluorescence photons are not scattered and the continuum emission photons are scattered once inside the cloud. Sgr B2 has a very complicated density profile, including three main compact cores (Sgr B2(N), Sgr B2(M), and Sgr B2(S) from north to south) and numerous clumpy regions. Overall, its density decreases as radius becomes larger. Without a model fully considering the density distribution within Sgr B2, the measured $\tau_{\mathrm{T}}$ for a specific region is an averaged value. For the $90^{\prime \prime}$ radius region we adopt and larger regions that previous works adopt (e.g., 4.5 radius region in Terrier et al. 2010), the 
averaged optical depth for such large regions is $\tau_{\mathrm{T}} \ll 1$; thus, it has been treated as optically thin as a whole. However, for the compact cores at the center of Sgr B2, which have higher local density, the local optical depths are higher than 1 as indicated by Herschel and NUSTAR measurements, though with large error bars. For these regions, multi-scattering could take place during the decay stage. The X-ray emission lifetime of optically thick material is longer than its light-crossing time $\left(t=\tau_{\mathrm{T}} r / c\right.$ when $\tau \gg 1$ ) owing to decay caused by multi-scattering. The reflected X-ray emission follows an exponential decay for optically thick materials and a parabolic decrease for optically thin materials. Therefore, the optically thicker material flux decreases much slower at late stages. If the compact core regions are confirmed to be optically thick, X-ray emission from the central compact cores Sgr B2(M) and Sgr B2(N) could still be bright enough to be detected when the surrounding optically thin regions completely fade. However, if the optical depth in the direction of the compact cores can be more tightly constrained to $\tau_{\mathrm{T}}=1$, the multi-scattering effect would be negligible. Future observation of Sgr B2 can test this prediction and better constrain the local optical depth in the direction of the compact cores.

\subsection{Cosmic-ray Electron Bombardment}

LECRe cannot be the major contribution to the bright $\mathrm{Fe} \mathrm{K} \alpha$ line emission from Sgr B2, as both the required iron abundance and the electron power are too high to be physical (e.g., Revnivtsev et al. 2004; Terrier et al. 2010). After a decade of decreasing in the $\mathrm{Fe} \mathrm{K} \alpha$ line flux, we tested whether the constraints on the LECRe parameters by the remaining emission level in 2013 are still excluding this scenario. Generating the bremsstrahlung emission with the observed slope of $\Gamma=1.9$ in $3-79 \mathrm{keV}$ requires relatively soft electron spectra with $s \sim 2.8$ and a minimum CR electron energy far below $100 \mathrm{keV}$ as we measured, which is illustrated in Tatischeff et al. (2012). For such a soft CR spectrum, the neutral $\mathrm{Fe} \mathrm{K} \alpha$ line is predicted to be relatively weak, with $\mathrm{EW}<0.4 \times\left(Z / Z_{\odot}\right) \mathrm{keV}$ (Figure 3 in Tatischeff et al. 2012). Therefore, to fit the observed Fe $\mathrm{K} \alpha$ line $\mathrm{EW}$ of $\sim 1.3 \mathrm{keV}$ as measured by $N U S T A R$, the required Fe abundance is found to be $Z_{\mathrm{Fe}}=4.0_{-0.6}^{+2.0}$, still significantly exceeding the current measurement of GC metallicity of solar to twice solar. This is due to the relatively inefficient production of $6.4 \mathrm{keV}$ photons by LECRe interactions, for which the Fe fluorescence yield $R_{6.4 \mathrm{keV}}=L_{\mathrm{X}}(6.4 \mathrm{keV}) /\left(d W_{e} / d t\right)$ is always lower than $1 \times 10^{-6}\left(Z / Z_{\odot}\right)$ for $s>2.3$ and $E_{\min }<100 \mathrm{keV}$ (Tatischeff et al. 2012). Besides the unphysical Fe abundance required by the $\mathrm{Fe} \mathrm{K} \alpha$ emission, the LECRe model also results in an overall poorer fitting to the broadband 3-79 $\mathrm{keV}$ spectrum compared to the XRN model. Especially, the hard X-ray part of the spectrum has more residuals for the LECRe model, thus providing a more exclusive constraint that does not depend on local metallicity.

Another difficulty in explaining all the $\mathrm{Fe} \mathrm{K} \alpha$ emission with LECRe is the lack of an obvious particle acceleration site. Yusef-Zadeh et al. (2002) suggested that the interaction of nonthermal radio filaments and the cloud could locally accelerate CR electrons. Yusef-Zadeh et al. (2007b) detected a nonthermal radio source in Sgr B2, but it is unclear whether it could serve as a particle accelerator. Even if there exists a local $\mathrm{CR}$ acceleration site, the nonthermal electrons with energies below $100 \mathrm{keV}$ are not able to escape the acceleration region and penetrate the whole dense cloud. Based on the unphysical values of the required metallicity and minimum electron energy, we conclude that the LECRe scenario cannot be a major contributor to the remaining X-ray emission from Sgr B2 in 2013. Currently, we do not have sufficient data to determine how much the LECRe bombardment may contribute to the $\mathrm{Sgr} \mathrm{B} 2 \mathrm{Fe} \mathrm{K} \alpha$ emission. Future X-ray polarization measurements could potentially reveal the contributions from X-ray reflection and CR bombardment.

\subsection{Cosmic-ray Ion Bombardment}

Although it is clear that the $\mathrm{Sgr} \mathrm{B} 2 \mathrm{Fe} \mathrm{K} \alpha$ emission decreases since 2001, the same level of the Fe $\mathrm{K} \alpha$ emission in 2012 and 2013 and the lack of measurements between 2005 and 2011 do not allow us to determine whether the $\mathrm{Fe} \mathrm{K} \alpha$ emission was still decreasing in 2013 or it has reached the constant background emission level. Future observations of Sgr B2 can distinguish between the two cases. Dogiel et al. (2009) estimated that CR protons could contribute to about $15 \%$ of the observed maximum $\mathrm{Fe} \mathrm{K} \alpha$ flux obtained around 2000, although this prediction is highly model dependent. In 2012 and 2013, the Sgr B2 Fe K $\alpha$ emission reached 20\% of that measured in 2001 (Section 4); therefore, the 2012 and $2013 \mathrm{Fe} \mathrm{K} \alpha$ emission could be mainly due to $\mathrm{CR}$ proton bombardment.

The 2013 NuSTAR Sgr B2 spectrum can be fit well with the self-consistent LECRp model. The required metallicity of $Z / Z_{\odot}=2.5_{-1.0}^{+1.5}$ overlaps with the GC environment metallicity of one solar to twice solar. Assuming that all the X-ray emission from the central 90" Sgr B2 in 2013 is due to CR proton bombardment, the required total power of the $\mathrm{CR}$ proton of energies between $E_{\min }=10 \mathrm{MeV}$ and $E_{\max }=1 \mathrm{GeV}$ in the cloud region is $d W / d t=(1.0 \pm 0.3) \times 10^{39} \mathrm{erg} \mathrm{s}^{-1}$. Taking the uncertainty of $E_{\min }$ into account, the required $\mathrm{CR}$ proton energy is in the range $d W / d t=(0.4-2.3) \times 10^{39} \mathrm{erg} \mathrm{s}^{-1}(\mathrm{Sec}-$ tion 5.1.4). According to Tatischeff et al. (2012), there is an additional $40 \%$ of power that comes from $\alpha$-particles with $C_{\alpha} / C_{p}=0.1$; the final required total kinetic $\mathrm{CR}$ ion power is $(0.6-3.2) \times 10^{39} \mathrm{erg} \mathrm{s}^{-1}$, which is roughly $10 \%$ of the steadystate mechanical power supplied by supernovae in the inner $\sim 200$ pc of the Galaxy (Tatischeff et al. 2012). The required power injection is quite significant and and probably difficult to fully be accounted with typical sources.

The power deposited into the cloud is lower than the incident $\mathrm{CR}$ ion power, because CR ions with energies lower than $E_{\text {min }}$ cannot penetrate the cloud and because, with a path length of $\Lambda=5 \times 10^{24} \mathrm{~cm}^{-2}$, those ions with energies higher than $180 \mathrm{MeV}$ can escape from the cloud without depositing energy in it (Tatischeff et al. 2012). Therefore, the power deposited by $\mathrm{CR}$ ions into central Sgr B2 is $\dot{W}_{d} \sim 8 \times 10^{38} \mathrm{erg} \mathrm{s}^{-1}$. With the central $90^{\prime \prime}$ of Sgr B2 mass of $M \sim(0.5-2) \times 10^{6} M_{\odot}$ estimated based on the simplified Sgr B2 density profile (Section 1) and a total mass of $M=6 \times 10^{6} M_{\odot}$ (Lis \& Goldsmith 1990), the corresponding ionization rate can be estimated to be $\zeta_{\mathrm{H}} \sim(6-10) \times 10^{-15} \mathrm{H}^{-1} \mathrm{~s}^{-1}$ using Equation (11) in Tatischeff et al. (2012). This CR ionization for the dense materials in Sgr B2 is comparable to the GC CR ionization rate of $\zeta_{\mathrm{H}} \sim(1-3) \times 10^{-15} \mathrm{~s}^{-1}$, which is found to be uniform throughout the GC on scales of 200 pc (e.g., Goto et al. 2011).

The GC LECR ion source has been suggested to be Galactic supernovae or star accretion onto Sgr A* (Dogiel et al. 2013 and the references therein). However, there could be local 
LECR ion sources, which would increase the local CR ionization rate and therefore the LECRp contribution to the X-ray emission (Dogiel et al. 2009; Tatischeff et al. 2012). The $\mathrm{X}$-ray emission from the Arches region used to be interpreted in this scenario. However, a significant decrease in the $\mathrm{Fe} \mathrm{K} \alpha$ line emission from the Arches revealed that the CR ion bombardment cannot be the dominant physical process (Clavel et al. 2014). Sgr B2 is by far the only GCMC that is likely to have reached or will soon reach the background X-ray emission level. Therefore, the remaining emission from Sgr B2 will be a unique powerful tool to probe the $\mathrm{CR}$ population in the $\mathrm{GC}$ in the X-ray energy bands. The LECRp scenario will stay valid as long as no further decrease is observed in the coming years.

\subsection{The Nature of $G 0.66-0.13$}

G0.66-0.13 is an elliptical feature with a major radius of $\sim 5 \mathrm{pc}$ and a minor radius of $\sim 3 \mathrm{pc}$. The illuminating source photon index $(\Gamma=1.4 \pm 0.5)$ is compatible with that of the central region of Sgr B2 $(\Gamma=2.2 \pm 0.4)$. The intrinsic column density of $N_{\mathrm{H}}(i)=3.0_{-1.9}^{+3.8} \times 10^{23} \mathrm{~cm}^{-2}$ is lower than the column density of the central part of Sgr B2. The primary source luminosity based on its 2012 peak $\mathrm{Fe} \mathrm{K} \alpha$ emission is $L_{8}=(1.3 \pm 0.5) \times 10^{38}(R / 100 \mathrm{pc})^{2}\left(Z_{\mathrm{Fe}}\right)^{-1} \mathrm{erg} \mathrm{s}^{-1}$, or $L_{3-79 \mathrm{keV}}=(4.3 \pm 1.6) \times 10^{38}(R / 100 \mathrm{pc})^{2}\left(Z_{\mathrm{Fe}}\right)^{-1} \mathrm{erg} \mathrm{s}^{-1}$.

It is consistent with the primary source luminosity required by the central region of Sgr B2. The Fe $\mathrm{K} \alpha$ line intensity of G0.66 -0.13 exhibits fast decay at $6.4 \mathrm{keV}$. The linear fitting shows that the G0.66-0.13 Fe $\mathrm{K} \alpha$ emission demonstrates an increasing trend prior to 2013 (Figure 5). However, the increase could be much sharper than what is shown in Figure 5. We do not have good enough statistics to distinguish between a linear increasing from 2000 to 2012 and a sharp peak in 2012 on top of a baseline Fe $\mathrm{K} \alpha$ emission. In 2012, the peak Fe $\mathrm{K} \alpha$ emission from G0.66-0.13 was brighter than the Sgr B2 core Sgr B2(M). The nondetection of its $\mathrm{Fe} \mathrm{K} \alpha$ emission in 2013 suggests a fast decrease in $\mathrm{Fe} \mathrm{K} \alpha$ line flux. The fast flux decrease with a lifetime of $t \sim 1 \mathrm{yr}$ roughly matches the lightcrossing time of the peak within G0.66-0.13 $(r / c \sim 2 \mathrm{yr})$.

Both Sgr B2 and G.66-0.13 are about 100 pc away from Sgr $A^{\star}$, while the G0.66-0.13 X-ray flux reached the peak 12-18 yr after Sgr B2. If G.66-0.13 was illuminated by the same Sgr A* outburst that illuminated Sgr B2, it should be 16-23 pc behind the Sgr B2 center in the line of sight assuming that Sgr B2 is $130 \mathrm{pc}$ in front of the projection plane (Reid et al. 2009). Therefore, G0.66-0.13 could be a molecular clump, with a higher local density than its surrounding environment, located in the Sgr B2 envelope, which extends to about $22.5 \mathrm{pc}$.

\section{CONCLUSIONS}

We studied the X-ray emission of the central $90^{\prime \prime}$ of Sgr B2 and a newly discovered cloud feature G0.66-0.13 with NUSTAR. We present their broadband spectra, morphology, and time variability and discuss two possible origins of the observed X-ray emission in 2013: X-ray reflection nebula and CR bombardment.

1. Central 90" of Sgr B2: The substructures of Sgr B2 are resolved at subarcminute scales at hard X-ray energy $(>10$ $\mathrm{keV})$. The hard X-ray continuum emission in $10-40 \mathrm{keV}$ reveals two compact star-forming cores Sgr B2(M) and Sgr B2 (N) surrounded by diffuse emission with the western side brighter than the eastern side. The central $90^{\prime \prime}$ region is marginally optically thin on average.

2. Main Compact Cores SgrB2(M) and SgrB2(N): Compact cores Sgr B2(M) and Sgr B2(N) are resolved above $10 \mathrm{keV}$ for the first time. Both the $6.4 \mathrm{keV}$ line emission and the $10-40 \mathrm{keV}$ continuum emission peak at the location of the main compact core Sgr B2(M). While Sgr B2(N) is significant in $10-40 \mathrm{keV}$ continuum emission, it is not detected at $6.4 \mathrm{keV}$ in 2013, perhaps owing to higher local absorption. The Sgr B2 (M) spectrum requires a high optical depth of $\tau_{\mathrm{T}}=1.3_{-1.0}^{+2.5}$, consistent with the Herschel measurement, suggesting that it is not optically thin.

3. XRN versus LECR: After a decade of decreasing, the 2012-2013 Fe K $\alpha$ emission reached $20 \%$ of that in 2001, but remained at the same level during 2012-2013. The lack of data between 2005 and 2011 does not allow us to determine whether the $2013 \mathrm{Fe} \mathrm{K} \alpha$ emission kept decreasing (which could be explained by the XRN or the LECRe model) or had reached the constant background level (for which the LECRp model is favored). We first excluded the LECRe scenario based on unphysical best-fit parameters, no matter whether the $\mathrm{Fe} \mathrm{K} \alpha$ emission is decreasing or not. The significant $6.4 \mathrm{keV}$ line of Sgr B2 in 2013 requires the Fe abundance to be at least 3.4 solar, significantly exceeding the current measurements of the GC metallicity. The best-fit minimum electron energy is far below $100 \mathrm{keV}$. With such low energies, the nonthermal electrons are not able to penetrate Sgr B2 even if there is a local CR particle acceleration site. Therefore, we conclude that the LECRe scenario cannot be a major contributor to the remaining level of the X-ray emission from Sgr B2 in 2013.

The 2013 Sgr B2 X-ray emission can be best explained by the XRN scenario if the X-ray emission is still decreasing. We examine the XRN scenario with an ad hoc XRN model and a self-consistent XRN model. Owing to the lack of geometrical information in this ad hoc model, interpretation of the intrinsic column density of $N_{\mathrm{H}}(i)=(5.0 \pm 1.2) \times 10^{23} \mathrm{~cm}^{-2}$ calls for caution. With the self-consistent XRN model MYTorus, we derive the intrinsic equatorial column density to be $N_{\mathrm{H}}(i)=(1.0 \pm 0.4) \times 10^{24} \mathrm{~cm}^{-2}$, twice that derived from the ad hoc model. This corresponds to $\tau_{\mathrm{T}}=0.67 \pm 0.27$, confirming that the central $90^{\prime \prime}$ region is marginally optically thin on average. In the XRN scenario, we develope an analytical method to calculate the primary source luminosity via Compton scattering with the $10-40 \mathrm{keV}$ scattered continuum (Equation (5)). With this approach the primary source luminosity in any $\mathrm{X}$-ray energy band can be derived with the observed continuum emission. The resulting primary source luminosity is $L_{3-79 \mathrm{keV}}(\mathrm{scat})=\left(5.6_{-2.9}^{+5.2}\right) \times 10^{38}(\mathrm{~d} / 100 \mathrm{pc})^{2} \mathrm{erg} \mathrm{s}^{-1}$. This is remarkably consistent with the primary source luminosity calculated via the photoelectric absorption process based on the updated source spectral shape, resulting in $L_{3-79 \mathrm{keV}}(\mathrm{abs})=$ $(5.0 \pm 2.3) \times 10^{38}(d / 100 \mathrm{pc})^{2} \mathrm{erg} \mathrm{s}^{-1}$. We find that $L_{8}(\mathrm{abs}) /$ $L_{8}$ (scat) is of order unity, confirming the self-consistency of the XRN model.

In case the $\mathrm{Fe} \mathrm{K} \alpha$ emission has reached the background level in 2013, the reflected X-rays could have completely faded and the LECRp process could be a major contributor. The required total $\mathrm{CR}$ ion power is $d W / d t=(0.6-3.2) \times 10^{39} \mathrm{erg} \mathrm{s}^{-1}$, about $10 \%$ of the mechanical power supplied by supernovae in the inner $\sim 200 \mathrm{pc}$ of the Galaxy. The CR ionization rate is found to be $\zeta_{\mathrm{H}} \sim(6-10) \times 10^{-15} \mathrm{H}^{-1} \mathrm{~s}^{-1}$, consistent with the $\mathrm{CR}$ ionization rate in the $\mathrm{GC}$ environment. If the Sgr B2 
$\mathrm{X}$-ray emission has indeed reached the background level, it would be a powerful tool to constrain the $\mathrm{CR}$ ion population in the GC.

4. The new cloud feature G0.66-0.13: The Fe $\mathrm{K} \alpha$ line flux of G0.66-0.13 reached its peak in 2012 and quickly diminished within $\sim 1$ yr. The fast variability is best explained by the XRN scenario. The required primary source luminosity $L_{3-79} \mathrm{keV}=$ $(4.3 \pm 1.6) \times 10^{38}(d / 100 \mathrm{pc})^{2} Z_{\mathrm{Fe}}^{-1} \mathrm{erg} \mathrm{s}^{-1}$ is consistent with that derived from central Sgr B2. Assuming that G.66-0.13 is illuminated by the same $\mathrm{Sgr} \mathrm{A}^{\star}$ outburst that illuminates Sgr B2, it should be 16-23 pc behind the Sgr B2 center along the line of sight, which is still within the Sgr B2 region. G0.66 -0.13 could be a molecular clump with a local column density higher than surroundings located in the Sgr B2 envelope.

5. Other GCMCs and Sgr $A^{\star}$ outburst history: In the NuSTAR Galactic plane survey, other GC giant molecular clouds were also detected well above $10 \mathrm{keV}$, including the "Bridge" and G0.13-0.13. The same methodology to derive the primary source luminosity is applied to each cloud and reconstructs the Sgr A* outburst history (Mori et al. 2015).

This work was supported under NASA Contract No. NNG08FD60C and made use of data from the NuSTAR mission, a project led by the California Institute of Technology, managed by the Jet Propulsion Laboratory, and funded by NASA. We thank the NuSTAR Operations, Software, and Calibration teams for support with the execution and analysis of these observations. This research has made use of the NuSTAR Data Analysis Software (NuSTARDAS) jointly developed by the ASI Science Data Center (ASDC, Italy) and the California Institute of Technology (USA). This research has also made use of data obtained with XMMNewton, an ESA science mission with instruments and contribution directly funded by ESA Member States and NASA. S.Z. is supported by NASA Headquarters under the NASA Earth and Space Science Fellowship Program-Grant "NNX13AM31." F.E.B. acknowledges support from CONICYT-Chile and the Ministry of Economy, Development, and Tourism's Millennium Science Initiative. G.P. acknowledges support via an EU Marie Curie Intra European fellowship under contract no. FP-PEOPLE-2012-IEF-331095 and Bundesministerium für Wirtschaft und Technologie/Deutsches Zentrum für Luft-und Raumfahrt (BMWI/DLR, FKZ 50 OR 1408) and the Max Planck Society. M.C., A.G., R.T., and S.S. acknowledge support by CNES.

\section{APPENDIX \\ APPLICABILITY OF MYTorus MODEL TO Sgr B2 SPECTROSCOPY}

The MYTorus model was originally developed to study $\mathrm{X}$-ray spectra of Compton-thick AGNs. The assumed geometry is a torus with a uniform distribution of neutral material reflecting incoming X-rays from an illuminating source at the center. Three model components are offered: the transmitted continuum (MYTZ), the scattered continuum (MYTS), and Fe fluorescence lines (MYTL). The model allows a range of values for three key model parameters: the illuminating source photon index $\Gamma=1.4-2.6$, the equatorial hydrogen column density (corresponding to the minor diameter of the torus) $N_{\mathrm{H}}=10^{22}-10^{25} \mathrm{~cm}^{-2}$, and the inclination angle $\theta_{\mathrm{obs}}=$ $0^{\circ}-90^{\circ}$. Considering a spherical molecular cloud as part of a virtual torus, the MYTorus model can be applied to molecular

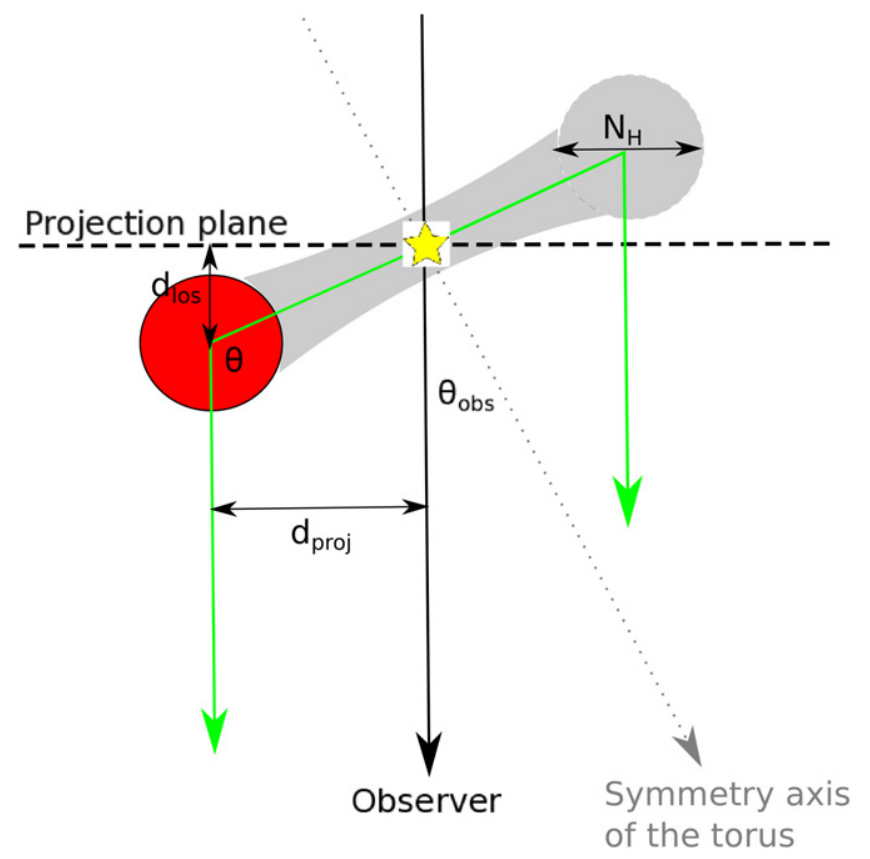

Figure 6. Spherical cloud (red circle) in a virtual torus (gray area) showing how the MyTorus model geometry is related to the GCMC geometry. The illuminating source (Sgr $\mathrm{A}^{\star}$ for GCMC) is shown by the yellow star. In the MYTorus model, the incident angle $\theta_{\text {obs }}$ is defined as the angle between the symmetry axis of the torus (dotted gray line) and the LOS (black vertical line). The equatorial column density $N_{\mathrm{H}}$ is defined over the minor diameter of the torus. When applying to the GCMC case, the quasi-spherical cloud can be considered as part of the torus. Incoming X-ray photons from the illuminating source are scattered by the cloud at an angle $\theta$ to the direction of the observer. The green lines show the travel paths of the X-ray photons. $d_{\text {proj }}$ is the projected distance between the cloud and the illuminating source as seen by the observer, and $d_{\text {los }}$ is the LOS distance between the cloud and the projected plane. A faceon view of the torus $\left(\theta_{\mathrm{obs}}=0^{\circ}\right)$ corresponds to the case where the cloud locates in the projected plane noted by a dashed black horizontal line $\left(\theta=90^{\circ}\right.$, $d_{\text {los }}=0$ ). The figure is from Appendix B1 in Mori et al. (submitted).

cloud spectra with some limitations. Figure 6 shows the geometry of a molecular cloud and a virtual torus. In the MYTorus model, the observation angle $\theta_{\text {obs }}$ is defined as the angle between the line of sight (LOS) and the symmetry axis of the torus. In contrast, most GCMC publications use the scattering angle $\theta$. A face-on case in the MYTorus model $\left(\theta_{\mathrm{obs}}=0^{\circ}\right)$ corresponds to a cloud in the same projected plane as the illuminating source, where the scattering angle is $\theta=90^{\circ}$. For three key assumptions of the MYTorus model, we discuss the valid parameter space where the model is applicable to the molecular cloud X-ray reflection spectra in the following.

\section{A.1. Toroidal Reflector}

In the face-on case $\left(\theta_{\mathrm{obs}}=0^{\circ}\right)$, MYTorus gives an accurate solution for a fully illuminated quasi-spherical molecular cloud, as different azimuthal parts of the torus all scatter at the same angle $\theta=90^{\circ}$. To derive the incoming $\mathrm{X}$-ray flux that illuminates the cloud (red circle in Figure 6, which is part of the gray virtual torus), we simply need to rescale it by the solid angle ratio of the torus (fixed to $\Omega / 2 \pi=0.5$ ) and the cloud. For Sgr B2, its solid angle with respect to Sgr $\mathrm{A}^{\star}$ is $\sim 2.89 \times 10^{-4}$. When the inclination angle deviates from $\theta_{\text {obs }}=0^{\circ}$, i.e., the face-on case, different azimuthal parts of the torus scatter incoming X-rays at different angles. The 
reflected spectrum thus shows variation and becomes inaccurate for a quasi-spherical molecular cloud. However, the scattered component MYTS does not vary strongly with $\theta_{\text {obs }}$ as long as $\theta_{\text {obs }} \lesssim 60^{\circ}$ and $N_{\mathrm{H}} \lesssim 10^{24} \mathrm{~cm}^{-2}$ (see Figure 17 in Mori et al. 2015). When $\theta_{\text {obs }}>60^{\circ}$, the spectral variation starts to become significant, for the following two reasons. First, as the inclination angle increases, the X-ray photons backscattered by the side of the torus hit the closer side of the torus before reaching the observer and thus are subject to further absorption. Second, multi-scattering can become important and cause angular-dependent X-ray flux, although it is negligible at $N_{\mathrm{H}} \lesssim 10^{24} \mathrm{~cm}^{-2}$.

Next, we determine the systematic error of measuring $N_{\mathrm{H}}, \Gamma$, and model normalization based on the MYTorus model. We simulated MYTS spectra for $\theta=60^{\circ}$ and $N_{\mathrm{H}}=10^{23}$ $10^{24} \mathrm{~cm}^{-2}$ and fit with the MYTS model with $\theta_{\mathrm{obs}}$ set to $0^{\circ}$. The deviations of the best-fit value of these model parameters from their input value are adopted as the systematic errors. At $N_{\mathrm{H}}=10^{23} \mathrm{~cm}^{-2}$, the deviations of $N_{\mathrm{H}}, \Gamma$, and model normalization from their input are $10 \%, 1 \%$, and $7 \%$, respectively, while at $N_{\mathrm{H}}=10^{24} \mathrm{~cm}^{-2}$, the deviations increase to $25 \%, 3 \%$, and $10 \%$. Therefore, we conclude that the reflected X-ray spectrum model MYTorus is not sensitive to the reflector geometry in the $N u S T A R$ energy band as long as $\theta_{\text {obs }} \lesssim 60^{\circ}$ and $N_{\mathrm{H}} \lesssim 10^{24} \mathrm{~cm}^{-2}$. Similar to the face-on case, the incident $\mathrm{X}$-ray flux can be derived by rescaling it with the solid angle ratio of the torus $\Omega / 4 \pi=0.5$ and central $90^{\prime \prime}$ of the Sgr B2 cloud $\Omega / 4 \pi=2.89 \times 10^{-4} \times(100 \mathrm{pc} / R)^{2}$ with systematic error $<10 \%$. However, the conversion from the incident angle in the MYTorus model to the position of the cloud cannot be well established. We note that the $N_{\mathrm{H}}$ measured by the MYTorus model is an averaged value over the torus: it does not take into account the specific geometry of the studied cloud and its possible partial illumination (see, e.g., Odaka et al. 2011). The systematic errors are estimated solely based on the MYTorus model and might therefore be underestimated. The more sophisticated model under development will better constrain the $N_{\mathrm{H}}$ (M. Walls et al. 2015, in preparation).

\section{A.2. Fe Abundance Fixed to Solar}

The Fe abundance is important in determining the incoming $\mathrm{X}$-ray flux solely from measurements of Fe fluorescent lines, while the $3-79 \mathrm{keV}$ broadband spectrum of NuSTAR also provides the energy range where Compton scattering dominates $(>10 \mathrm{keV})$ over Fe fluorescence. The incoming X-ray flux is determined consistently from both the Compton scattering process with the MYTS component and the Fe fluorescence process via the MYTL component. The best-fit model parameters $\left(N_{\mathrm{H}}, \Gamma\right.$, and normalization) of MYTS do not vary significantly when the MYTS model is fit to the cloud X-ray spectra with or without the $6-10 \mathrm{keV}$ energy range that covers the $6.4 \mathrm{keV} \mathrm{Fe} \mathrm{K} \alpha$ line, the $7.06 \mathrm{keV} \mathrm{Fe} \mathrm{K} \beta$ line, and the $7.1 \mathrm{keV} \mathrm{Fe} \mathrm{K}$ edge. Data tables for different $\mathrm{Fe}$ abundances in the range of $Z_{\mathrm{Fe}}=0.5-3.0$ will be implemented to the modified XRN model for molecular clouds.

\section{A.3. Uniform Density}

The incoming X-ray flux from the illuminating source will not be significantly affected by nonuniform density profiles as long as $N_{\mathrm{H}} \leqslant 10^{24} \mathrm{~cm}^{-2}$, where multi-scattering is negligible.
If the density is not uniform, large clouds can include very dense clumps where multi-scattering effects can be significant. For Sgr B2, the largest optical depth is $\tau_{\mathrm{T}} \sim 1$ in the direction of the densest cores Sgr B2(M), in which case the multiscattering effects are negligible. The current XRN model does not address the complicated density profile of Sgr B2, which contains compact cores, clumps, and an overall decreasing density profile. A more sophisticated XRN model with a reasonable density profile of Sgr B2 implemented is under development (M. Walls et al. in preparation) and will reduce the uncertainties caused by the nonuniformity of Sgr B2.

\section{REFERENCES}

Anders, E., \& Grevesse, N. 1989, GeCoA, 53, 197

Baganoff, F. K., Bautz, M. W., Brandt, W. N., et al. 2001, Natur, 413, 45

Baganoff, F. K., Maeda, Y., Morris, M., et al. 2003, ApJ, 591, 891

Balucinska-Church, M., \& McCammon, D. 1992, ApJ, 400, 699

Bambynek, W., Crasemann, B., Fink, R. W., et al. 1974, RvMP, 46, 853

Barrière, N. M., Tomsick, J. A., Baganoff, F. K., et al. 2014, ApJ, 786, 46

Benson, J. M., \& Johnston, K. J. 1984, ApJ, 277, 181

Bernard, J. P., Paradis, D., Marshall, D. J., et al. 2010, A\&A, 518, L88

Capelli, R., Warwick, R. S., Porquet, D., Gillessen, S., \& Predehl, P. 2012, A\&A, 545, A35

Clavel, M., Soldi, S., Terrier, R., et al. 2014, MNRAS, 443, L129

Clavel, M., Terrier, R., Goldwurm, A., et al. 2013, A\&A, 558, A32

Cunha, K., Smith, V. V., Sellgren, K., et al. 2008, in IAU Symp. 245, Formation and Evolution of Galaxy Bulges, ed. M. Bureau, E. Athanassoula \& B. Barbuy (Cambridge: Cambridge Univ. Press), 339 Davies, B., Origlia, L., Kudritzki, R.-P., et al. 2009, ApJ, 694, 46

de Vicente, P., Martin-Pintado, J., \& Wilson, T. L. 1997, A\&A, 320, 957

Dogiel, V. A., Chernyshov, D., Yuasa, T., et al. 2009, PASJ, 61, 1093

Dogiel, V. A., Chernyshov, D. O., Kiselev, A. M., et al. 2014, APh, 54, 33

Dogiel, V. A., Chernyshov, D. O., Tatischeff, V., et al. 2013, ApJL, 771, L43

Etxaluze, M., Goicoechea, J. R., Cernicharo, J., et al. 2013, A\&A, 556, A137

Ghez, A. M., Salim, S., Weinberg, N. N., et al. 2008, ApJ, 689, 1044 Gillessen, S., Eisenhauer, F., Trippe, S., et al. 2009, ApJ, 692, 1075

Giveon, U., Sternberg, A., Lutz, D., Feuchtgruber, H., \& Pauldrach, A. W. A 2002, ApJ, 566, 880

Goto, M., Usuda, T., Geballe, T. R., et al. 2011, PASJ, 63, L13

Harrison, F. A., Craig, W. W., Christensen, F. E., et al. 2013, ApJ, 770, 103 Inui, T., Koyama, K., Matsumoto, H., \& Tsuru, T. G. 2009, PASJ, 61, 241

Koyama, K., Inui, T., Hyodo, Y., et al. 2007, PASJ, 59, 221

Koyama, K., Maeda, Y., Sonobe, T., et al. 1996, PASJ, 48, 249

Krivonos, R. A., Tomsick, J. A., Bauer, F. E., et al. 2014, ApJ, 781, 107

Lis, D. C., \& Goldsmith, P. F. 1990, ApJ, 356, 195

Madsen, K. K., Harrison, F. A., Markwardt, C. B., et al. 2015, ApJS, 220, 8

Molina, M., Bassani, L., Malizia, A., et al. 2013, MNRAS, 433, 1687

Molinari, S., Bally, J., Noriega-Crespo, A., et al. 2011, ApJL, 735, L33

Mori, K., Hailey, C. J., Krivonos, R., et al. 2015, ApJ, 814, 94

Morris, M., Ghez, A. M., \& Becklin, E. E. 1999, AdSpR, 23, 959

Morris, M., \& Serabyn, E. 1996, ARA\&A, 34, 645

Muno, M. P., Baganoff, F. K., Brandt, W. N., Park, S., \& Morris, M. R. 2007, ApJL, 656, L69

Muno, M. P., Bauer, F. E., Baganoff, F. K., et al. 2009, ApJS, 181, 110

Murakami, H., Koyama, K., \& Maeda, Y. 2001, ApJ, 558, 687

Murphy, K. D., \& Yaqoob, T. 2009, MNRAS, 397, 1549

Nobukawa, M., Ryu, S. G., Tsuru, T. G., \& Koyama, K. 2011, ApJL, 739, L52

Nowak, M. A., Neilsen, J., Markoff, S. B., et al. 2012, ApJ, 759, 95

Odaka, H., Aharonian, F., Watanabe, S., et al. 2011, ApJ, 740, 103

Ponti, G., Morris, M. R., \& Clavel, M. 2014, in IAU Symp. Vol. 303, The Galactic Center: Feeding and Feedback in a Normal Galactic Nucleus, ed. L. O. Sjouwerman, C. C. Lang \& J. Ott (Cambridge: Cambridge Univ. Press), 333

Ponti, G., Morris, M. R., Terrier, R., et al. 2015, MNRAS, 453, 172

Ponti, G., Morris, M. R., Terrier, R., \& Goldwurm, A. 2013, in Advances in Solid State Physics, Vol. 34, ed. D. F. Torres \& O. Reimer, 331

Ponti, G., Terrier, R., Goldwurm, A., Belanger, G., \& Trap, G. 2010, ApJ, 714, 732

Porquet, D., Grosso, N., Predehl, P., et al. 2008, A\&A, 488, 549 
Reid, M. J., Menten, K. M., Zheng, X. W., et al. 2009, ApJ, 700, 137

Revnivtsev, M. G., Churazov, E. M., Sazonov, S. Y., et al. 2004, A\&A, 425, L49

Ross, R. R., \& Fabian, A. C. 2005, MNRAS, 358, 211

Sato, F., Hasegawa, T., Whiteoak, J. B., et al. 2000, ApJ, 535, 857

Snowden, S. L., Mushotzky, R. F., Kuntz, K. D., et al. 2008, A\&A, 478,615

Sunyaev, R., \& Churazov, E. 1998, MNRAS, 297, 1279

Sunyaev, R. A., Markevitch, M., \& Pavlinsky, M. 1993, ApJ, 407, 606

Takagi, S. i., Murakami, H., \& Koyama, K. 2002, ApJ, 573, 275
Tatischeff, V., Decourchelle, A., \& Maurin, G. 2012, A\&A, 546, A88

Terrier, R., Ponti, G., Bélanger, G., et al. 2010, ApJ, 719, 143

Valinia, A., Tatischeff, V., Arnaud, K., Ebisawa, K., \& Ramaty, R. 2000, ApJ, 543, 733

Yusef-Zadeh, F., Hewitt, J. W., Wardle, M., et al. 2013, ApJ, 762, 33

Yusef-Zadeh, F., Law, C., \& Wardle, M. 2002, ApJL, 568, L121

Yusef-Zadeh, F., Muno, M., Wardle, M., \& Lis, D. C. 2007a, ApJ, 656, 847

Yusef-Zadeh, F., Wardle, M., \& Roy, S. 2007b, ApJL, 665, L123

Zhang, S., Hailey, C. J., Baganoff, F. K., et al. 2014, ApJ, 784, 6 